\title{
A Review and Prospect for the Complexity and Resilience of Urban Public Transit Network Based on Complex Network Theory
}

\author{
Lin Zhang, ${ }^{1,2,3}$ Jian Lu $\mathbb{D}^{1,2,3}$ Bai-bai Fu, ${ }^{4}$ and Shu-bin Li ${ }^{5}$ \\ ${ }^{1}$ Jiangsu Key Laboratory of Urban ITS, Southeast University, Nanjing 211189, China \\ ${ }^{2}$ Jiangsu Province Collaborative Innovation Center of Modern Urban Traffic Technologies, Southeast University, Nanjing 211189, China \\ ${ }^{3}$ School of Transportation, Southeast University, Nanjing 211189, China \\ ${ }^{4}$ School of Architecture and Urban Planning, Shandong Jianzhu University, Jinan 250101, China \\ ${ }^{5}$ Department of Traffic Management Engineering, Shandong Police College, Jinan 250014, China
}

Correspondence should be addressed to Jian Lu; lujian_1972@seu.edu.cn

Received 17 September 2018; Accepted 14 November 2018; Published 4 December 2018

Academic Editor: Massimiliano Zanin

Copyright (C) 2018 Lin Zhang et al. This is an open access article distributed under the Creative Commons Attribution License, which permits unrestricted use, distribution, and reproduction in any medium, provided the original work is properly cited.

\begin{abstract}
The complexity and resilience of urban public transit network (PTN) are the interdisciplinary study area between transportation engineering and system science, which is a good demonstration of applying complex network theory to promote the development of engineering science. The deep understanding of this study helps to provide a new perspective for analyzing the reliability of urban PTN. Following study process of the complexity and resilience of complex network, this paper reviews the complexity and resilience of PTN from four topics, i.e., the PTN complexity, the static resilience of PTN, the dynamic resilience (cascading failures based resilience) of single layered PTN, and the dynamic resilience of interdependent PTN. In the literature review, multiple key items are, respectively, extracted for each topic, and the engineering applicability of each topic is also analyzed, which are both for obtaining the key features of this study area. Finally, in order to realize the development trend of cyclic and forward-complex network theory, network resilience theory, transforming into a realistic model and method that is close to actual public transit operation, engineering application and practice, and contributing to complex network theory, the study status is summarized and the future development trend is prospected.
\end{abstract}

\section{Introduction}

Traffic congestion, parking problem, and car pollution are becoming the main bottleneck restricting the sustainable development of cities. The advanced traffic management experiences of Zurich, Curitiba, and Singapore [1], as well as the implementation experience of national traffic unimpeded project in China, have shown that the one effective strategy for alleviating urban traffic congestion problem is to prioritize the development of multimodes public transit network (PTN). This trend makes the "public transit plus nonmotorized mode" be considered as the optimal trip mode, making a contribution to transform the traditional traffic mode into a green and low-carbon mode. "Public transit plus nonmotorized mode" is essentially the "core trip plus last kilometer trip"; thus the reliability of core trip mode (i.e., reliability of public transit) is widely concerned by scholars. However, the studies on the reliability of public transit system are mainly focused on the service reliability, such as the headway reliability $[2,3]$ and the running time reliability $[4,5]$. In other words, the studies on system reliability which is based on network topological structure are ignored. As a kind of system topological structure reliability, the basic theory of PTN resilience originates from complex network theory, providing a new perspective for analyzing the PTN reliability.

Since 1990s, there is a popular trend to study the basic theories of complexity and resilience of complex network, which is inspired by Watts's work about small-world network 
[6], Barabasi's work about scale-free network [7], and Albert's work about resilience of complex network [8]. The introduction of complex network theory provides a new perspective for multidisciplinary scholars and promotes the development of related interdisciplinary subjects, such as power grids [9, $10]$, social science $[11,12]$, and transportation engineering [1316]. The complex network resilience is classified as the static resilience and the dynamic resilience (it also denotes the cascading failures in this paper, i.e., the cascading failures based resilience) by recognizing whether the failure node or edge contributes to the failure of adjacent nodes or edges. With the rapid development of computer, information, and control technologies, the connections among various real world networks are closely related, and the interdependent and interaction features become more apparent. Benefited from a deep understanding of this trend, the work of Buldyrev et al. [17] further advanced the study on complex network resilience into an interesting and novel perspective, i.e., the complex network resilience under an interdependent perspective.

PTN is analyzed as a typical complex network; thus it also follows the aforementioned development process. Unfortunately, the studies on complexity and resilience of PTN based on complex network theory generally have a misunderstanding; i.e., the topological structure study is considered as the supremacy, while the actual PTN operation characteristics are not considered enough. Because of this misunderstanding, the previous studies lack the engineering applicability and cannot significantly improve the PTN reliability. In particular, urban PTN mentioned in this paper is the multimodes PTN, and its priority development is considered as one effective strategy for alleviating urban traffic congestion problem. In other words, it includes the bus transit network (BTN), tram transit network, and urban rail transit network (URTN, it is general designation of subway, urban light rail transit) which all provide the public transit serve for urban internal residents trip, but not the air transportation network, national railway network, national coach network, etc. Additionally, it also should be noted that, in the study area of complex network resilience, there is a confusing use among the resilience, vulnerability, and robustness concepts, let alone in the study area of complex transportation network. Mattsson et al. [18] illustrated the distinction between these concepts and thought that it is meaningful and useful to make this distinction, but it is not possible to draw a precise boundary. For clarifying the sameness and difference among three concepts, Reggiani et al. [19] reviewed emerging concepts of resilience and vulnerability in transportation network related studies. Moreover, they also found that both the resilience and robustness concepts seem to be used with a similar meaning in various transportation networks. Thus, a fine distinction has been made; i.e., the vulnerability is more about the susceptibility of a complex network and the resilience concerns more with the response of a complex network. Nevertheless, they are actually used in a mix, such as the resilience concept used in the works of Berche et al. [20] and Leu et al. [21], the robustness concept used in the works of Yang et al. [22] and Gu et al. [23], and the vulnerability concept used in the works of Sun et al. [24] and Bao et al. [25]. On the one hand, these aforementioned examples all fall within the study framework of static resilience of PTN with no fundamental difference. On the other hand, the cascading failures model seems to be much closer to the resilience concept. Consequently, this paper uses the resilience concept for reviewing the topic that covers both static resilience and dynamic resilience of PTN.

However, the other transportation networks that are connected to urban PTN via several special stations should also be paid attention, because the large integrated passenger station or the integrated transportation station is found in more and more cities that have an expanding city size. In other words, there is a growing trend to have a clear and close connection between the urban PTN and other transportation networks. Because of the urban PTN as the core component of urban integrated transportation network and a similar analytical framework existing in various transportation networks, the complexity and resilience of urban PTN are reviewed in the following text, while the complexity and resilience of other transportation networks can be seen in the pioneer reviews of Reggiani et al. [19, 26] and Mattsson et al. [18]. The complexity and resilience of air transportation network especially should be paid more attention, such as the pioneer review of Zanin et al. [16] and the related studies of Neal et al. [15], Wandelt et al. [27], Sun et al. [28, 29], and Cardillo et al. [30]. As a result, the key features of urban PTN based on complex network theory can be obtained through focusing on perspective of engineering applicability; especially they are extracted from numerous transportation network studies. Additionally, some static resilience related works of PTN are not reviewed in this paper, because they are not strictly the complex network theory related studies, such as the serviceability based resilience of PTN (RodríguezNúñez et al. [31]). Moreover, the cascading failures based resilience models are usually regarded as the mesoscopic reliability model, because the redistributed passenger flow on each edge is essentially the aggregated passenger flow of multiple routes, not just one single bus vehicle's passenger flow, such as the work of Zhang et al. [32]. Thus, some dynamic resilience related works of PTN are also not reviewed in this paper, because they are not the cascading failures based resilience related studies of PTN, such as the vehicle scale based microcosmic resilience model (Cats et al. [33]).

The main contributions of this paper can be summarized as follows: for promoting the engineering application of complex network approach of urban PTN, this paper reviews the complexity and resilience of PTN from four topics based on interdisciplinary study perspectives between transportation engineering and system science, i.e., the PTN complexity, the static resilience of PTN, the dynamic resilience of single layered PTN, and the dynamic resilience of interdependent PTN. Additionally, multiple key items are, respectively, extracted for each topic, and the engineering applicability of each topic is also analyzed, which are both for obtaining the key features of this study area. Finally, the study status is summarized and the future development trend is prospected, so as to provide some references for relevant researchers.

The remainder of this paper is organized as follows: Section 2 provides the review and analysis of the PTN complexity. Section 3 provides review and analysis of the static 
resilience of PTN. Section 4 provides review and analysis of the dynamic resilience of single layered PTN. The review and analysis of dynamic resilience of interdependent PTN is presented in Section 5. Finally, Section 6 contains the summaries and prospects.

\section{PTN Complexity}

2.1. Review. Urban PTN is usually analyzed as a complex and dynamic giant system composed of road system, flow system, and management service system. Combined with its complex passenger travel selection behavior, urban PTN is significantly different from the general complex network and is considered as a complex combination of technical network and social network. Study on the PTN complexity based on complex network statistical physics provides a new perspective for understanding urban public transit system that is synthetically influenced by socioeconomic activities, geospatial features, cultural customs, public policies, etc. To accurately understand the complex essence of urban PTN, this paper summarizes the studies on complex topological characteristics of PTN from seven aspects: study time, case city, PTN type/whether multilayered systems are considered, modeling method, network node size, complexity indicator, and major contribution or conclusion, as shown in Table 1. In particular, the major contributions or conclusions listed the different parts of various references in the review table, not the overly similar references.

2.2. Analysis. Through reviewing the studies on PTN complexity, we can find that the study time is concentrated on 2002 to 2018, and the scholars have applied complex network theory to conduct extensive empirical studies on the complex topological characteristics of urban PTN, involving many cities. It should be noted that these studies are the basis for studying the extended problems of complex PTN. This study area is still in the process of continuous development and is the frontier area of complex network study, which presents the following features.

(a) The modeling methods of PTN are used for extracting various types of information. Different methods may be implemented by attributing different constituents of the real world network to graph nodes and edges. Currently, the most commonly used modeling methods of complex PTN have been further developed into a mature modeling system, including the Space L method (see the example in the works of Sienkiewicz et al. [38], Ferber et al. [40], etc.), the Space P method (see the example in the works of Sienkiewicz et al. [38], Ferber et al. [40], etc.), and the Space R method (see the example in the works of Ferber et al. [40], Zhang et al. [67], etc.).

(i) With the Space L representation method, stations are represented by nodes. If two stations (stations $i$ and $j$ ) are sequentially gone through by one or more common routes and adjacent to each other (i.e., without station in between), there is an edge between two stations with $a_{i j}^{l}=1$ in the corresponding adjacency matrix $A_{L}$; otherwise $a_{i j}^{l}=0$, and define $a_{i i}^{l}=0$. (ii) With the Space P representation method, stations are represented by nodes. If station $i$ can directly link station $j$ by one single route (no matter two stations are adjacent or not), there is an edge between two stations with $a_{i j}^{p}=1$ in the corresponding adjacency matrix $A_{P}$; otherwise $a_{i j}^{p}=0$, and define $a_{i i}^{p}=0$.

(iii) With the Space $\mathrm{R}$ representation method, the routes are represented by nodes. If two routes (routes $i$ and $j$ ) have at least one intersecting (transfer) station, there is an edge between two nodes (routes) with $a_{i j}^{r}=1$ in the corresponding adjacency matrix $A_{R}$; otherwise $a_{i j}^{r}=0$, and define $a_{i i}^{r}=0$.

In particular, identification of Space L method, Space $\mathrm{P}$ method, and Space $\mathrm{R}$ method is a start only, but not sufficient. When the weights of passenger flow (Soh et al. [49], Xu et al. [63], etc.), the actual distance between stations that is calculated by spatial coordinates embedding (Zhen et al. [54], Yang et al. [65], etc.), the travel time between two adjacent stations (Alessandretti et al. [60]), the number of routes passing through two adjacent stations (Zhao et al. [39], Sui et al. [52], etc.), the number of same stations between two routes (Xu et al. [41], $\mathrm{Lu}$ et al. [43], etc.), and the departure frequency of public transit (Zhang et al. [69], etc.) are considered, there are corresponding modified modeling methods, namely, modified Space L method, modified Space P method, and modified Space R method. Moreover, it also should be noted that a modified Space L method, namely, the supernode graph method, is proposed in the work of Shanmukhappa et al. [68]. The set of nodes (stations) whose geographic distances calculated by spatial coordinates embedding are within 100 meters are combined to represent a single node called supernode. Additionally, the presence of points of interests (POIs, such as a hospital, hotel, office, school, shopping complex, residential apartment, etc.) around the station and the number of routes passing through two adjacent stations are simultaneously considered as the weights in their work. In a nutshell, these modified modeling methods introduce the influences of various weights for providing more realistic information on PTN.

(b) The significant impacts of multilayered systems on the overall network properties have been shown in the subway network and BTN of Boston (Latora et al. [34]). Thus, whether multilayered systems are considered is our particular concern. Analyzing the item of "PTN type/whether multilayered systems are considered" (see Table 1), it mostly focuses on one single type selected from bus and urban rail transit, or one single layer selected from public transit geographical network layer, public transit transfer network layer, and public transit route network layer. Although the multilayered public transit systems are considered in early works of this study area (Latora et al. [34], Ferber et al. [37], Sienkiewicz et al. [38], Ferber et al. [40], Ferber et al. [47], Berche et al. [48], etc.), the various public transit types do not form a strict coupled network or interdependent network. The multimodes are not considered as possessing significant differences with each other; i.e., the nodes of bus and the nodes of urban rail transit are not strictly 


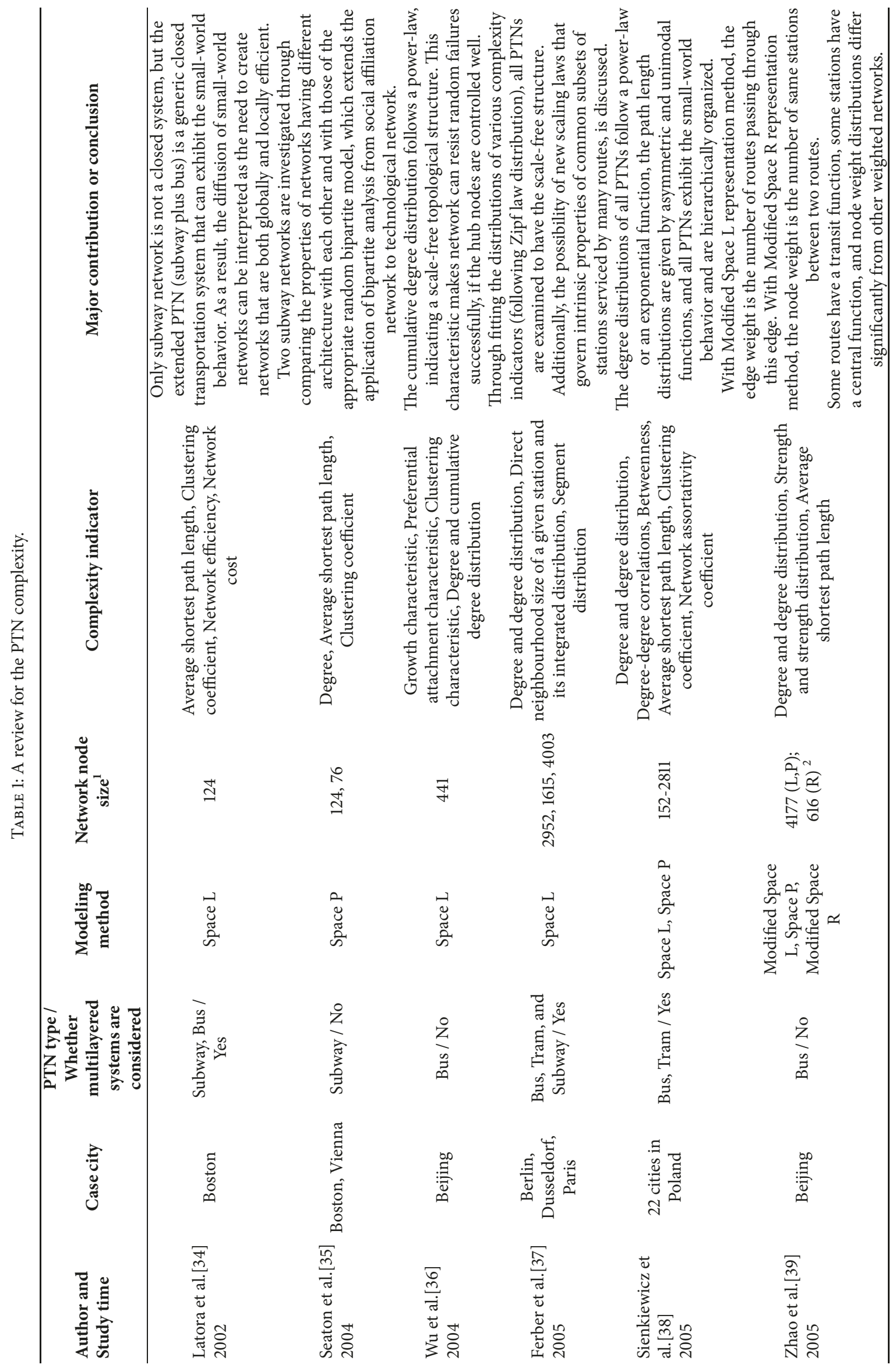




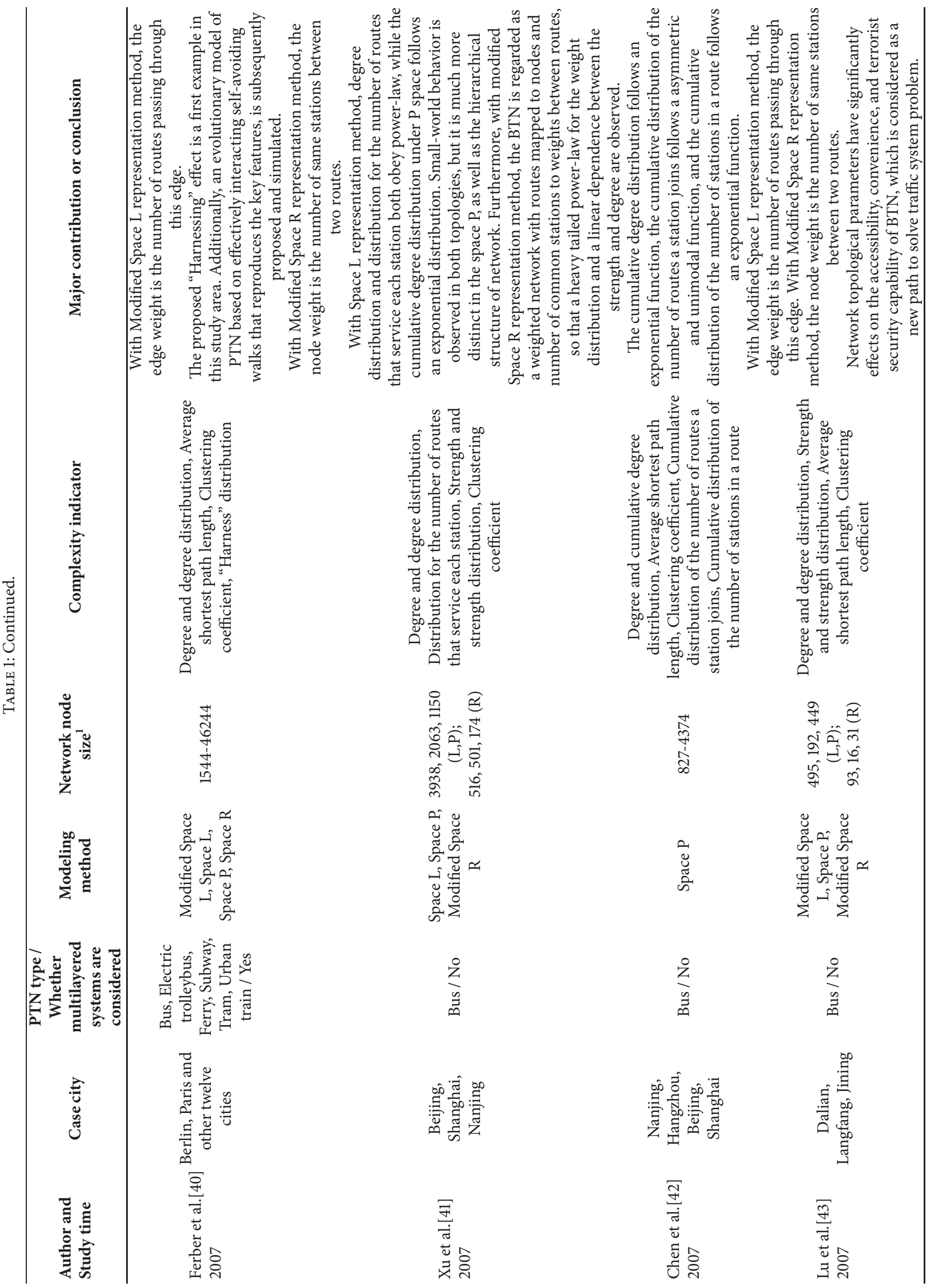




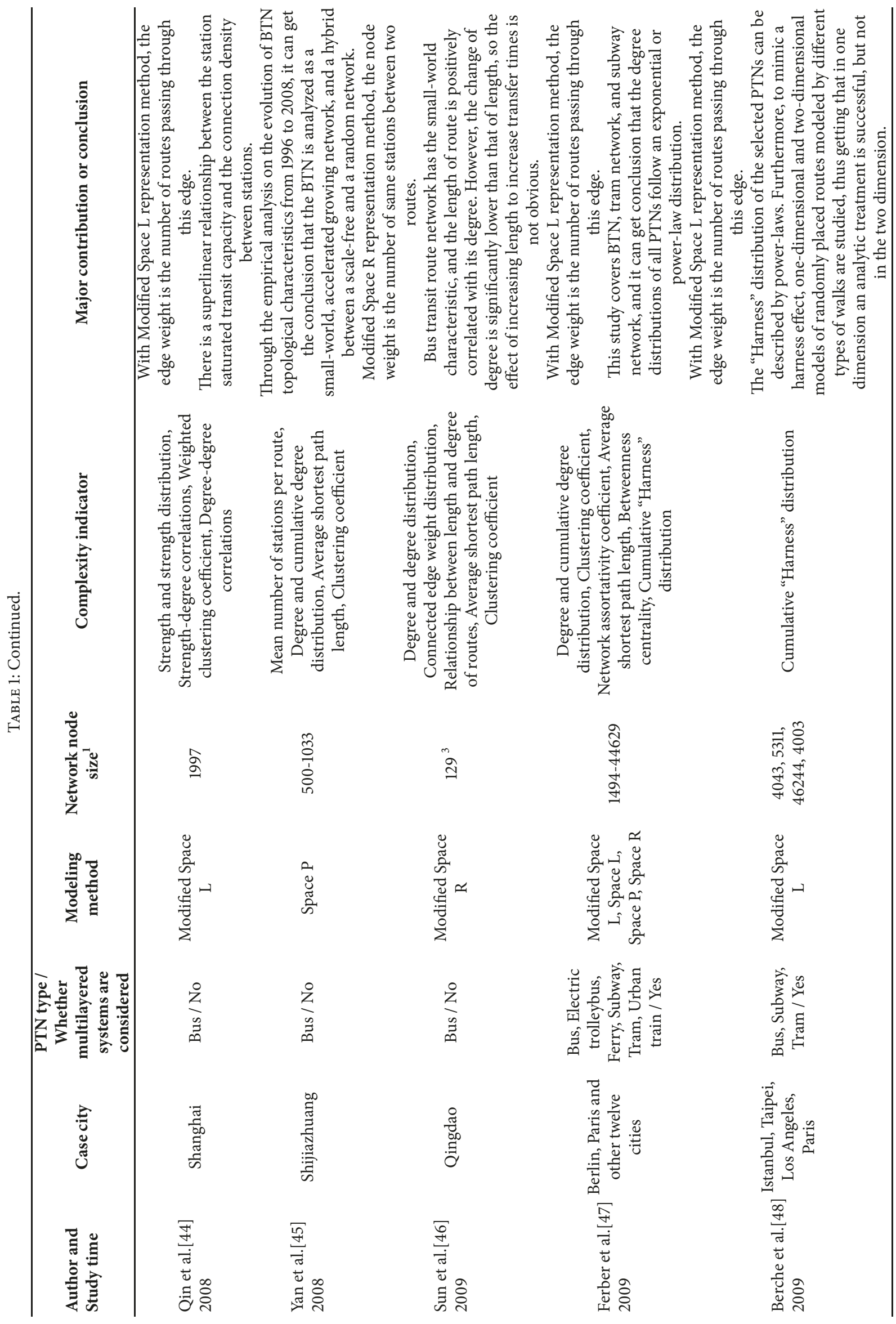




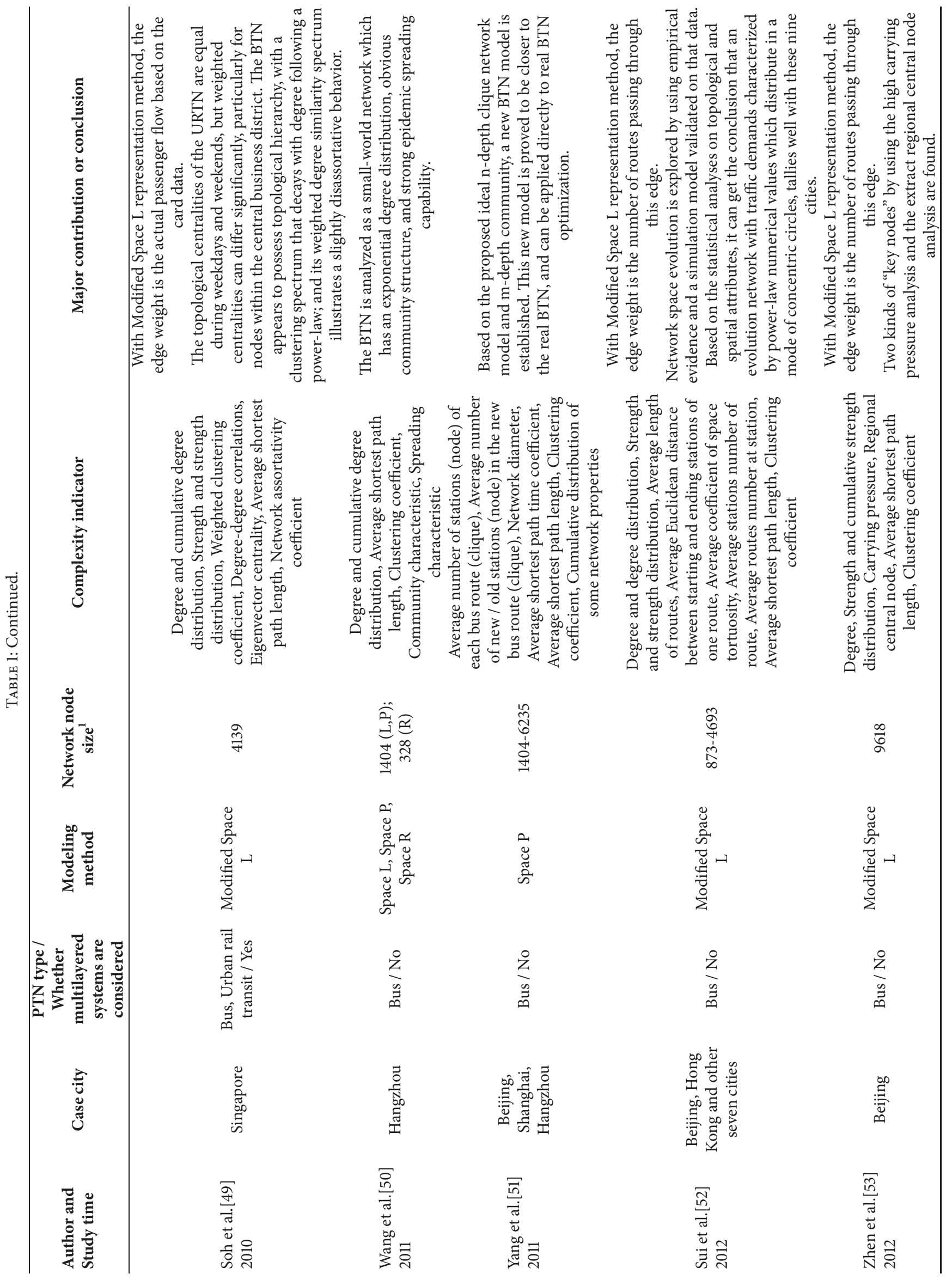




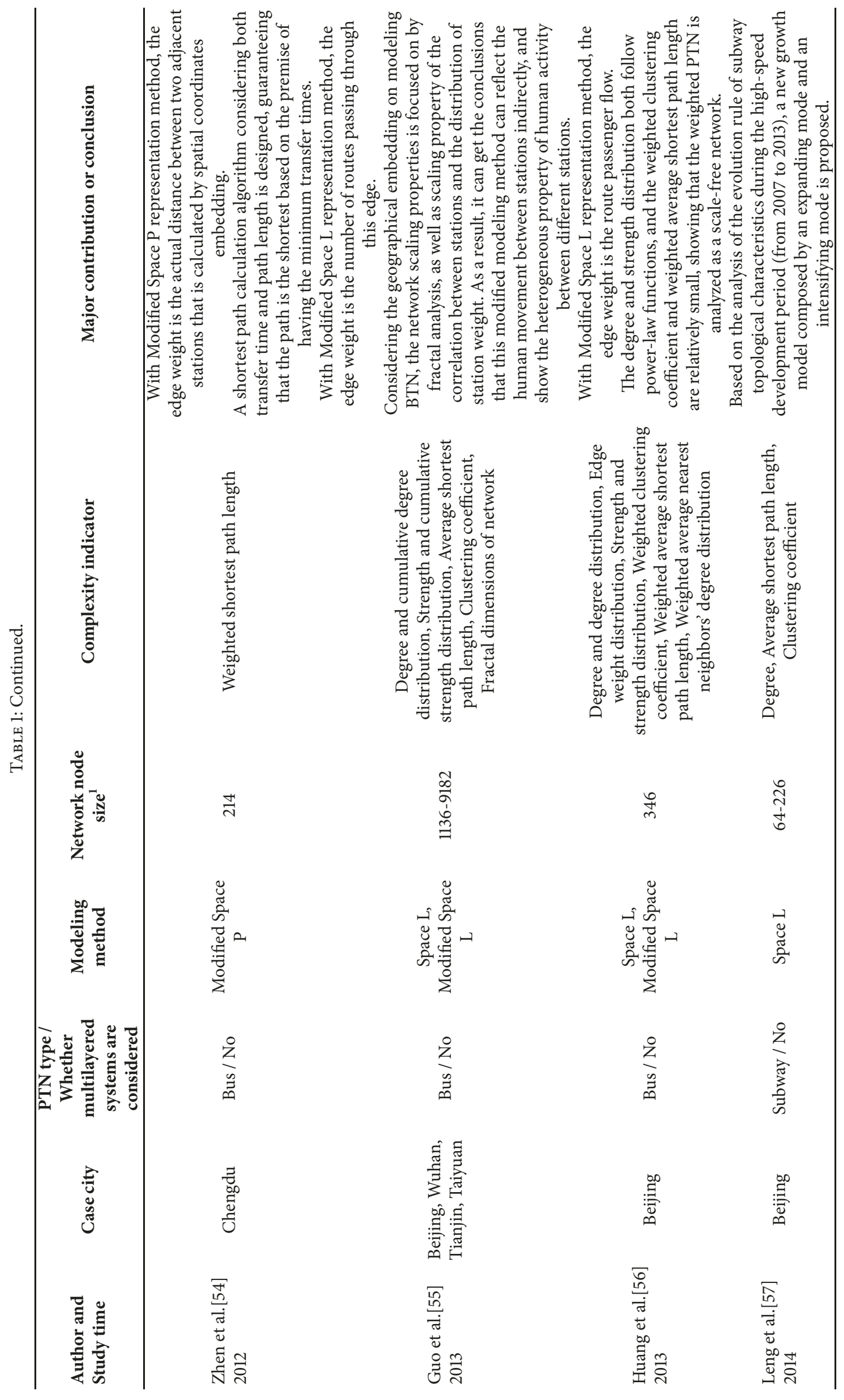




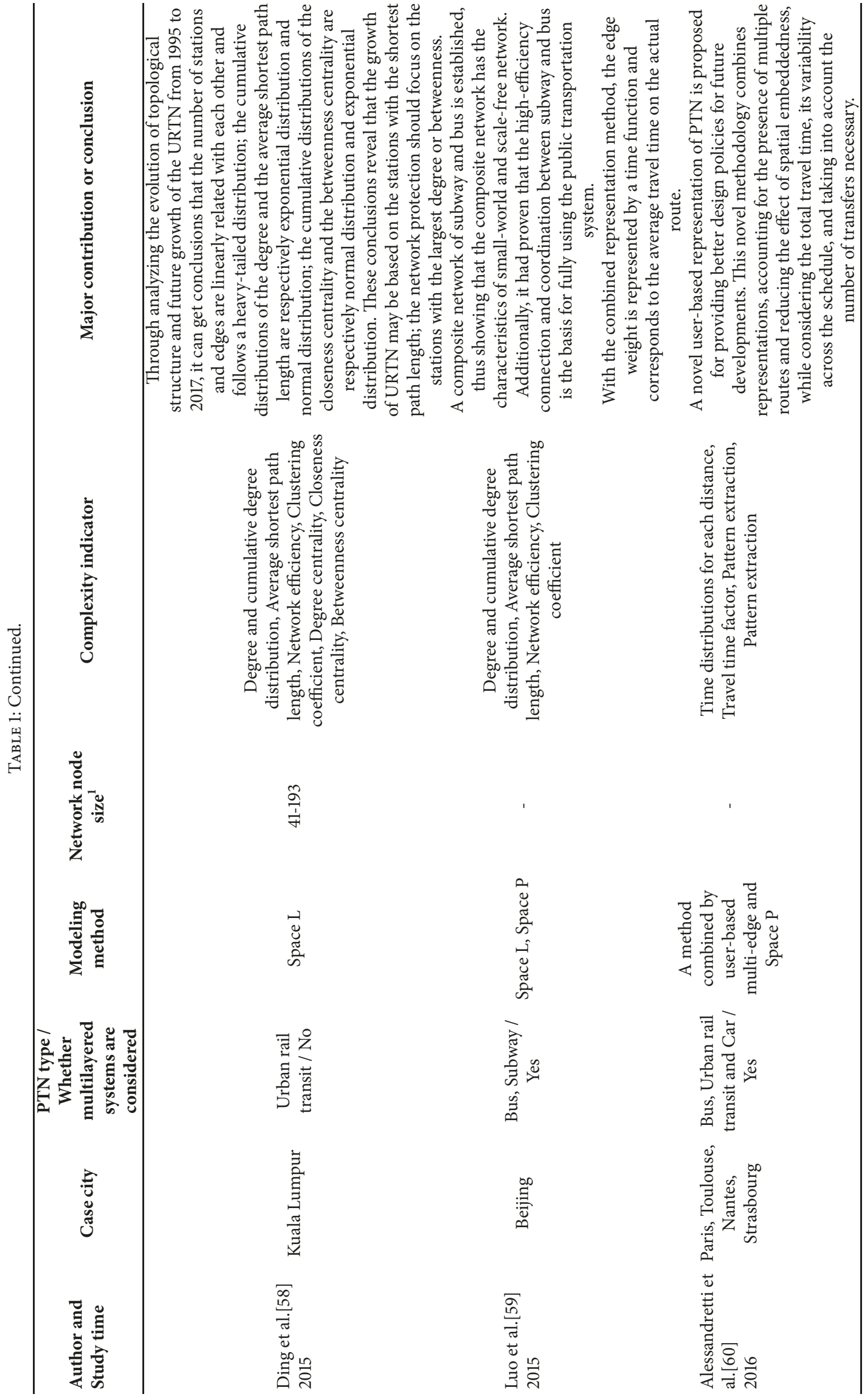




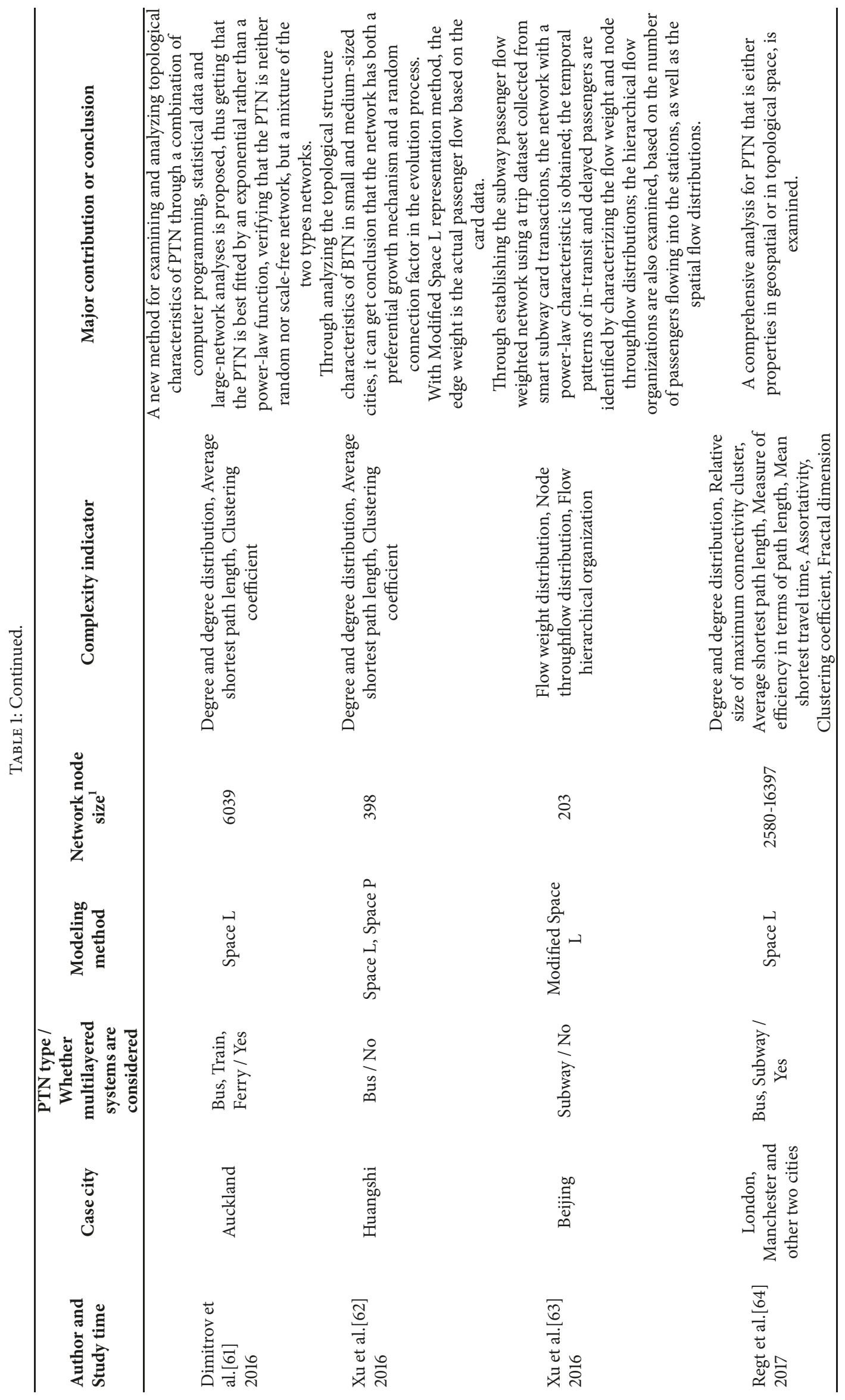




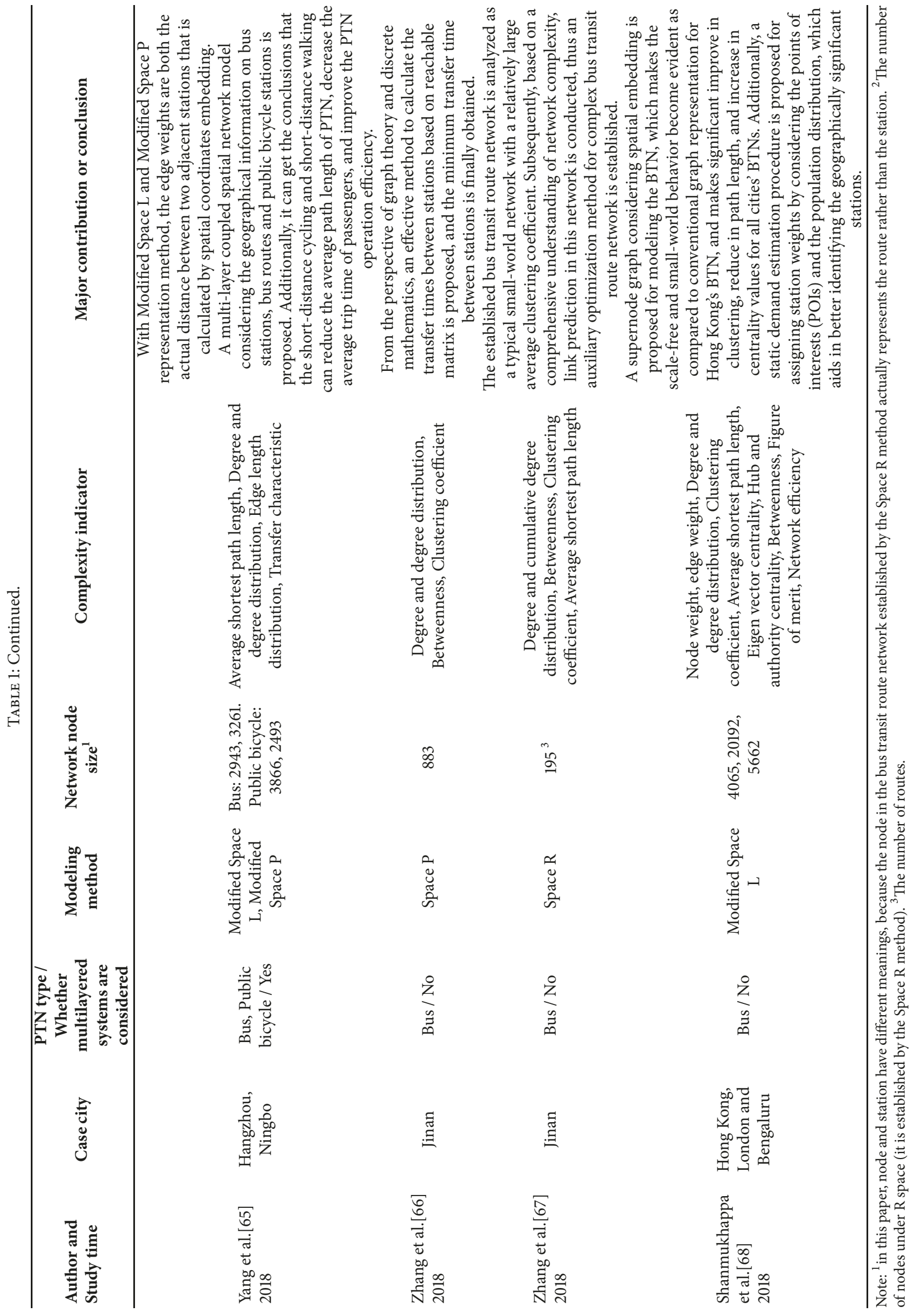


differentiated when the multilayered public transit system is represented into a network model. Additionally, the nodes of bus and the nodes of urban rail transit do not have a clear interdependent type or coupled method. Subsequently, each type of multilayered systems is, respectively, analyzed and compared in the work of Soh et al. [49], or the stations with consistent name are treated as one node of represented network model in the work of Luo et al. [59]. However, the composite PTN (or multimodes PTN) still does not form a strict coupled network or interdependent network, because the clear coupled standard between bus station and urban rail transit station is not proposed. In particular, the same trend exists in the other study line; i.e., the studies on various layers of a single type of PTNs established by different network modeling methods also have this problem (Zhao et al. [39], Ferber et al. [40], Xu et al. [41], etc.).

Recently, the study has been developed from the complexity of single type or single layer PTN into the complexity of multitype or multilayer composite PTN. In a nutshell, these novel trends have enriched the study scale and dimension. The multitypes or multilayers of public transit system are truly considered as a coupled system or an interdependent system. It should be noted that these trends open the frontier study area which is considered to establishing the PTN in a more realistic way, i.e., the complex PTN under interdependent perspective (the detailed introduction is shown in Section 5, dynamic resilience of interdependent PTN). As a frontier study network type, its modeling method is essentially a combination of the aforementioned six network modeling methods (mentioned in Section 2.2 (a)), which are generally divided into two categories:

(i) One is that the study objects are the various layers of a single type of PTN (e.g., BTN). The number of nodes in each layer network is the same and corresponds to one another, such as an interdependent PTN combined by the public transit geographical network layer and the public transit transfer network layer. (see the detailed process in the works of Dong et al. [70], Zhang et al. [71], etc.)

(ii) The other one is that the study objects are two types of PTNs (e.g., BTN and URTN). The number of nodes in two different types of PTNs is obviously different, and most of them rely on the complementary relationship between geographical location and function to form an interdependent PTN. (see the detailed process in the works of Yang et al. [72], Huang et al. [73], etc.)

(c) In most studies, the most important process is to identify the network topology type through analyzing the degree distribution or cumulative degree distribution. Then, through fitting an exponential distribution or a power-law distribution and combining with the analyses of clustering coefficient (usually a large value) and average shortest path length (usually a small value), it can get that the network exhibits the small-world behavior or scale-free characteristic (Latora et al. [34], Sienkiewicz et al. [38], Xu et al. [41], etc.), and even the hybrid between a scale-free and a random network (Yan et al. [45], Dimitrov et al. [61]).
The complexity indicators are becoming more comprehensive and their computational complexities are increasing day by day, which can better reveal the interaction between the topological structure and function of urban PTN (see this trend in Table 1, complexity indicator). More specifically, it initially focuses on the basic topological parameters such as degree and degree distribution, betweenness, clustering coefficient, and average shortest path length, etc. Subsequently, it has developed to focus on the developed complexity indicators such as community characteristic, spreading characteristic, network assortativity coefficient, and carrying pressure. This progress makes it possible to deeply understand complex PTN based on the comprehensive and multilevels complexity indicators. Additionally, it also provides a necessary analysis basis for the extended studies of multiscales dynamic evolution, link prediction, cascading failures, synchronization, and seepage of complex PTN, e.g., the nodes (or edges) with larger degree, betweenness, strength are all the key types of nodes (or edges) in the PTN, so they are usually taken as the various attack targets in the simulation of PTN resilience (see the detailed association relationship in the Section 3.2 (b)).

In particular, a specific indicator that is considered as being closer to the perspective of engineering applicability than the other existing complexity indicators is proposed. This specific indicator, called the "harness" (Ferber et al. [40], Ferber et al. [47], Berche et al. [48], etc.), provides an example for using the complexity indicators to better understand the complex PTN in a more realistic way. More specifically, it is defined under consideration of the phenomenon that bus routes sharing the same grid of streets and tracks are often found to proceed in parallel along shorter or longer sequences of stations. Network "harness" is used to quantify this phenomenon that is calculated through sequences of stations serviced by each route. Additionally, a similar feature has been treated in weighted BTN (Xu et al. [41]). It should be noted that both methods produce the interesting phenomena; i.e., they both follow the power-law distribution. Another interesting application is that the extent to which harness characteristic is expressed may obviously play a role for the attack resilience of a PTN (Berche et al. [48]). Although the evidence is very limited, such a result may be still expected, because the bus route which does not share the same streets is more resilient. In other words, the networks with weaker "harness" characteristic are more resilient. This specific indicator has a certain connection with a similar concept in urban bus transit planning project, i.e., the repetition coefficient of route. In actual bus transit planning practice, traffic engineers always try their best to reduce the repetition coefficient of bus route. Maybe this connection is the bridge between the complex network theory and transportation engineering technology. Therefore, a wider application and validation of "harness" should be conducted in the PTN of various cities.

(d) Other analyses for better capturing the main features of this study area are summarized below.

(i) The spatial embedding is recognized as an important operation for gaining useful insights into resilience and efficiency of PTN. However, only small part of 
works (Zhen et al. [54], Yang et al. [65], Regt et al. [64], etc.) takes it into account, due to the lack of available data on spatial coordinates of PTN. On the one hand, as mentioned in Section 2.2 (a), the actual distance between stations that is calculated by spatial coordinates embedding (Zhen et al. [54], Yang et al. [65], etc.) can be weighted to establish the PTN model or resilience model accurately. This point is critical to making these models be close to the reality. On the other hand, it is closely related to the fractal analysis (Guo et al. [55], Regt et al. [64]). It should be noted that the studies on fractal analysis of urban PTN are not all listed in Table 1, because the fractal analysis framework is quite different from the current analysis framework based on complex network theory (summarized by this paper in Table 1, including multiple key items). An important fact is that there have been far less of fractal studies when compared to topological studies. The first example of a fractal object to illustrate the concept of fractal is taken from geography: coastlines are, in general, fractals (Mandelbrot et al. [74]). Recently, the notion of a fractal is summarized in the work of Regt et al. [64]; i.e., it is often used to quantify development and growth of cities and their communication and transportation systems. For the related studies on PTN, it mainly concerns the density of stations or the total length of track as a function of the distance from the center of a network (Benguigui et al. [75], Kim et al. [76], etc.), as well as the distributions of interstation distances of consecutive stations (Ferber et al. [77]).

(ii) The study on the PTN complexity is gradually approaching the practical engineering application. In other words, the developing trend from theory into practice and the developing trend from system science into transportation engineering are both becoming a reality. More specifically, on the one hand, the empirical analysis of the PTN complexity has mostly concentrated on the topology analysis of unweighted network, emphasizing the study conducted on the basis of the physical nature. Subsequently, it has developed to establish the weighted PTN considering the effects of geography distance between stations, passenger flow, and departure frequency, making the description of the PTN complexity close to actual PTN operation. It should be noted that the significances of weights had been realized by Latora et al. [34] at the beginning of this study area: the weighted networks providing more realistic information on modeling various PTNs. And this key problem is further developed with the proposed modified methods for PTN, which has been discussed in Section 2.2(a). On the other hand, it mostly analyzes the PTN complexity in a fixed year. Subsequently, it has developed to analyze the evolution rule of network complexity under long-term scale, which is conducive to provide data accumulation by establishing some correlation between the complexity indicators of PTN and the network growth model (Yan et al. [45], Leng et al. [57], and Ding et al. [58]).

(iii) The connection between the PTN complexity and its derivative issues is clearer to expand the basic study scale of complex PTN; e.g., in terms of the connection between the complexity and link prediction, bus transit route network of Jinan city is analyzed as a typical small-world network with a large clustering coefficient. This feature indicates that the structural similarity-based link prediction will show a good performance in this network. Thus, inspired by the missing (new) link prediction and the spurious existing link identification in link prediction theory, an auxiliary optimization method for bus transit route network is established, which can be used not only to optimize current BTN but also to evaluate BTN planning (Zhang et al. [67]).

\section{Static Resilience of PTN}

3.1. Review. The study on the resilience of PTN is based on a comprehensive understanding of the PTN complexity, and it is classified as the static resilience and the dynamic resilience by recognizing whether the failure station or edge contributes to the failure of adjacent stations or edges. With the increasing number of terror attacks on PTN, such as the subway explosion of Moscow in 2004 and the subway and bus explosion of London in 2005, the government administrators and scholars have paid more attention to the PTN reliability that is relevant to the coordinated operation and public working life of urban transport systems. This endeavor is expected to provide decision support for the management and operation of PTN. This paper summarizes the studies on the static resilience of PTN from six aspects: study time, case city, PTN type/whether multilayered systems are considered, resilience measurement indicator, attack strategy, and major contribution, or conclusion, as shown in Table 2.

3.2. Analysis. Through reviewing the studies on static resilience of PTN, we can find that the study time is concentrated on 2006 to 2018, and the scholars have applied complex network theory to conduct extensive case studies on the static resilience of PTN, involving many cities. This study area is still a hot area of complex network study, which presents the following features.

(a) As Ferber et al. [82] pointed out in their work, the impact on complex network behavior upon removing either nodes or edges is essentially related to the lattice percolation phenomena (see the detailed introduction in the work of Stauffer et al. [93]). The study on the static resilience of PTN is based on the extension of the study framework of complex network resilience given by Albert et al. [8]. Through conducting an empirical analysis on multiple scale-free real world networks, it can find that they display a unexpectedly high resilience under random attacks $[94,95]$. However, if they face the deliberate attacks that target important nodes or edges, they may appear to be especially vulnerable [96, 97]. The same evidences also existed in the PTN, because 


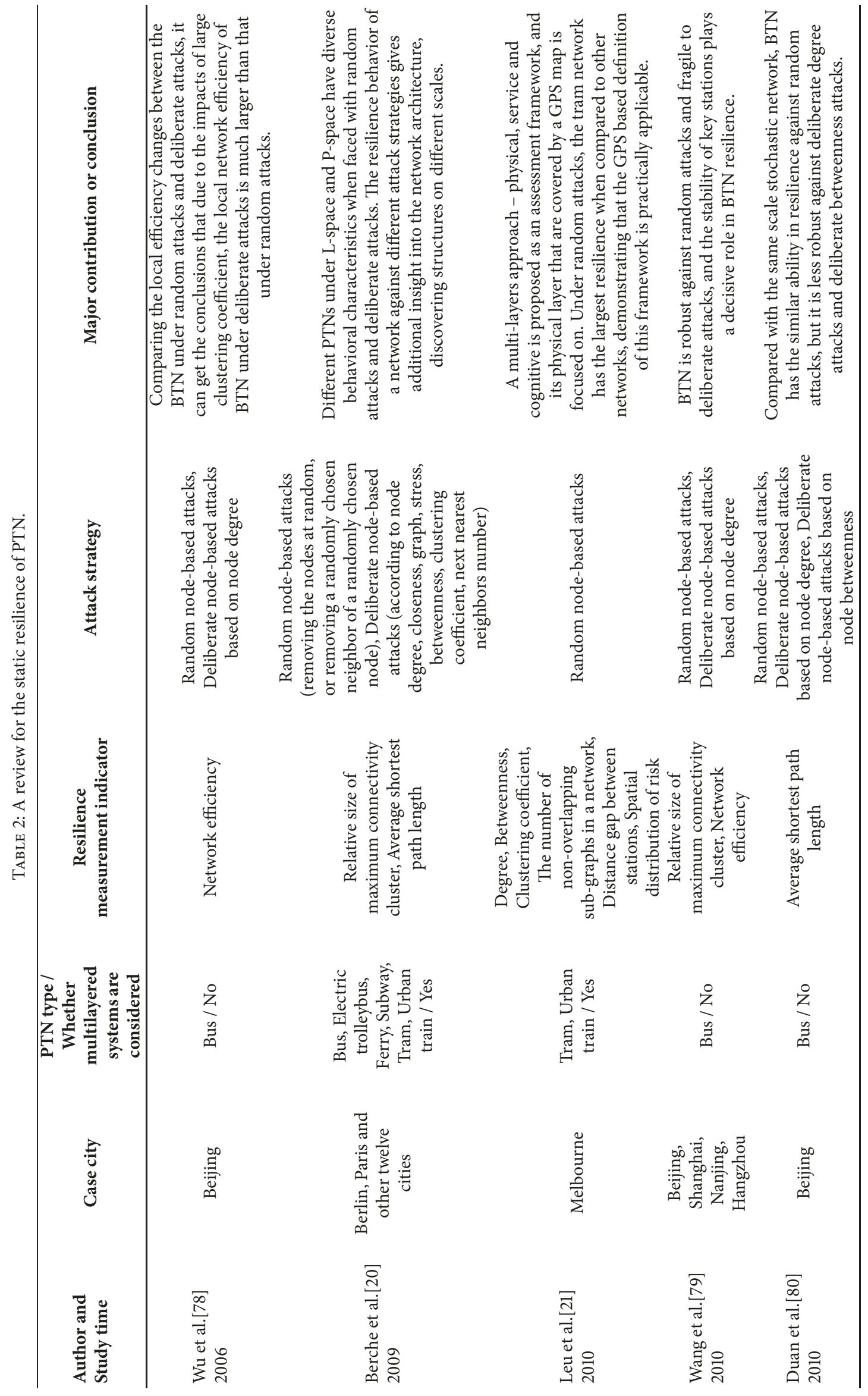




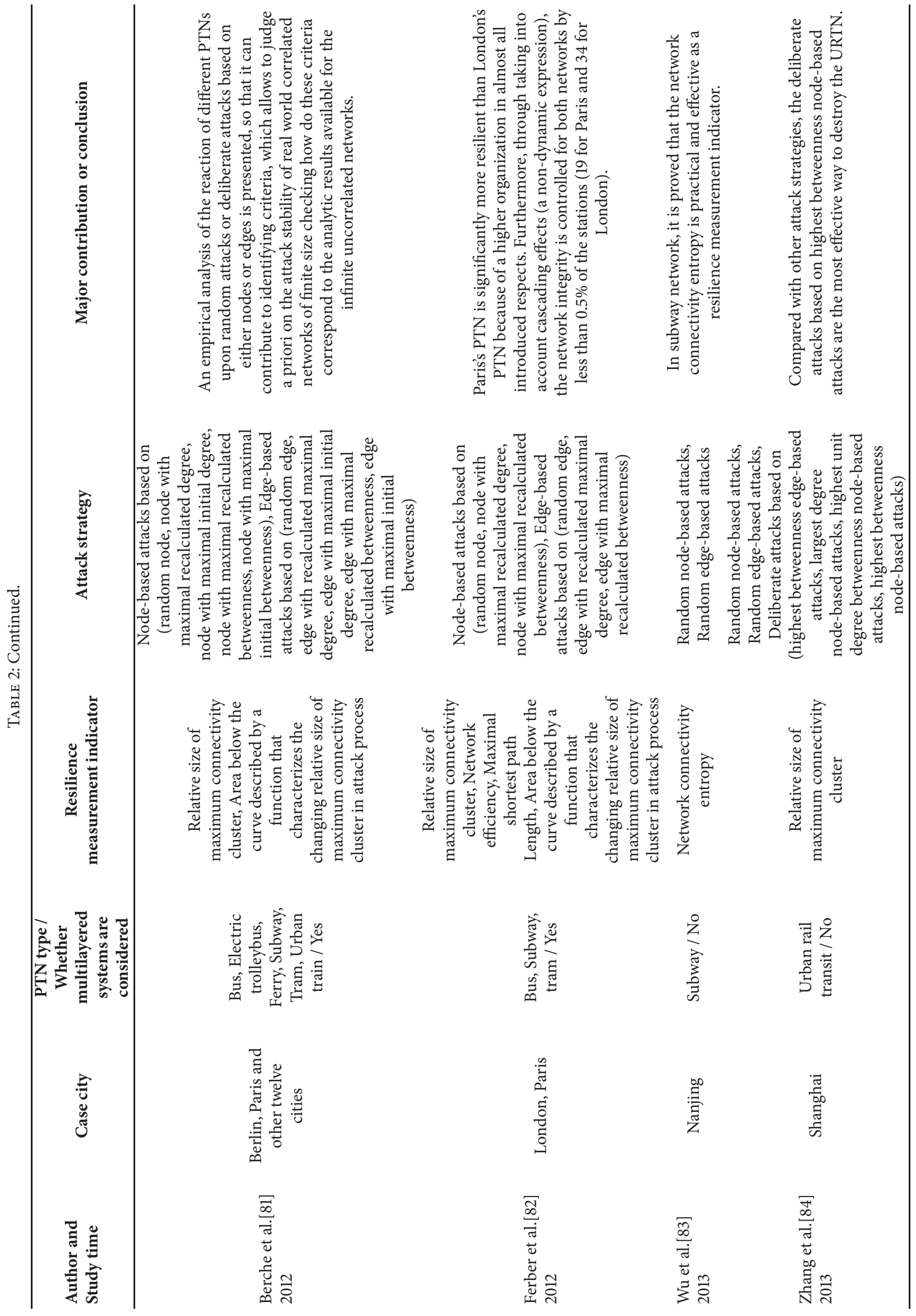




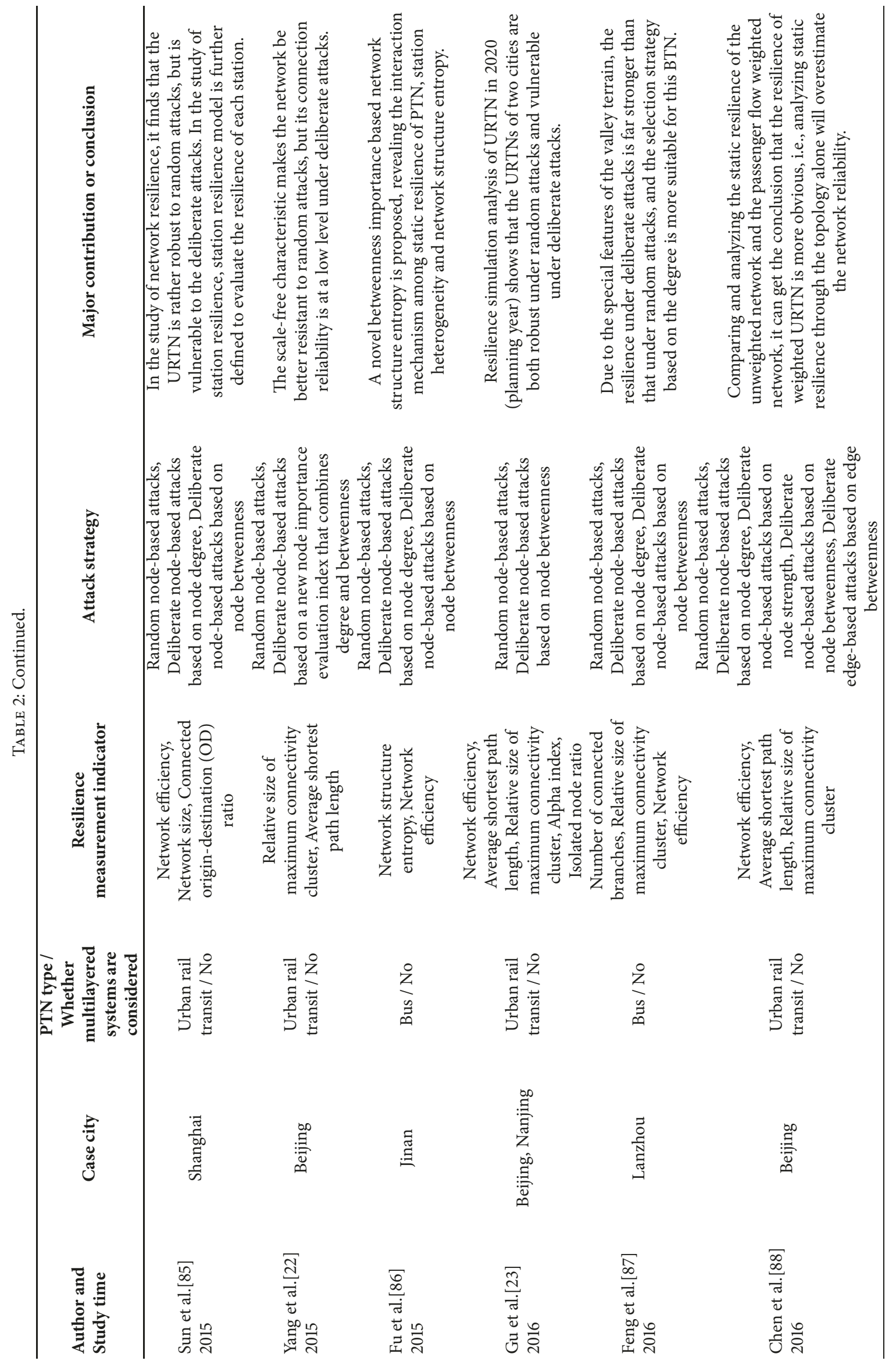




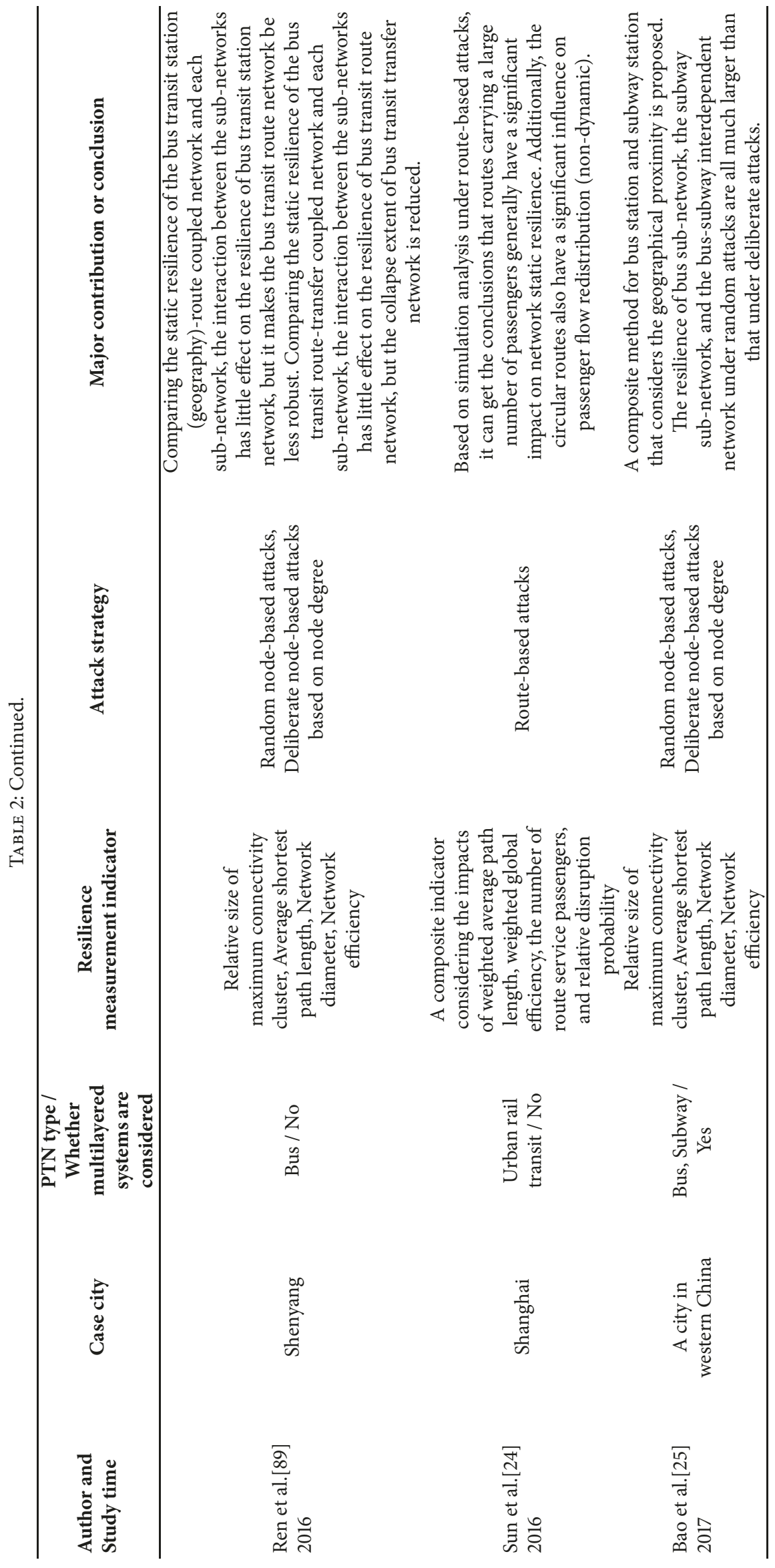




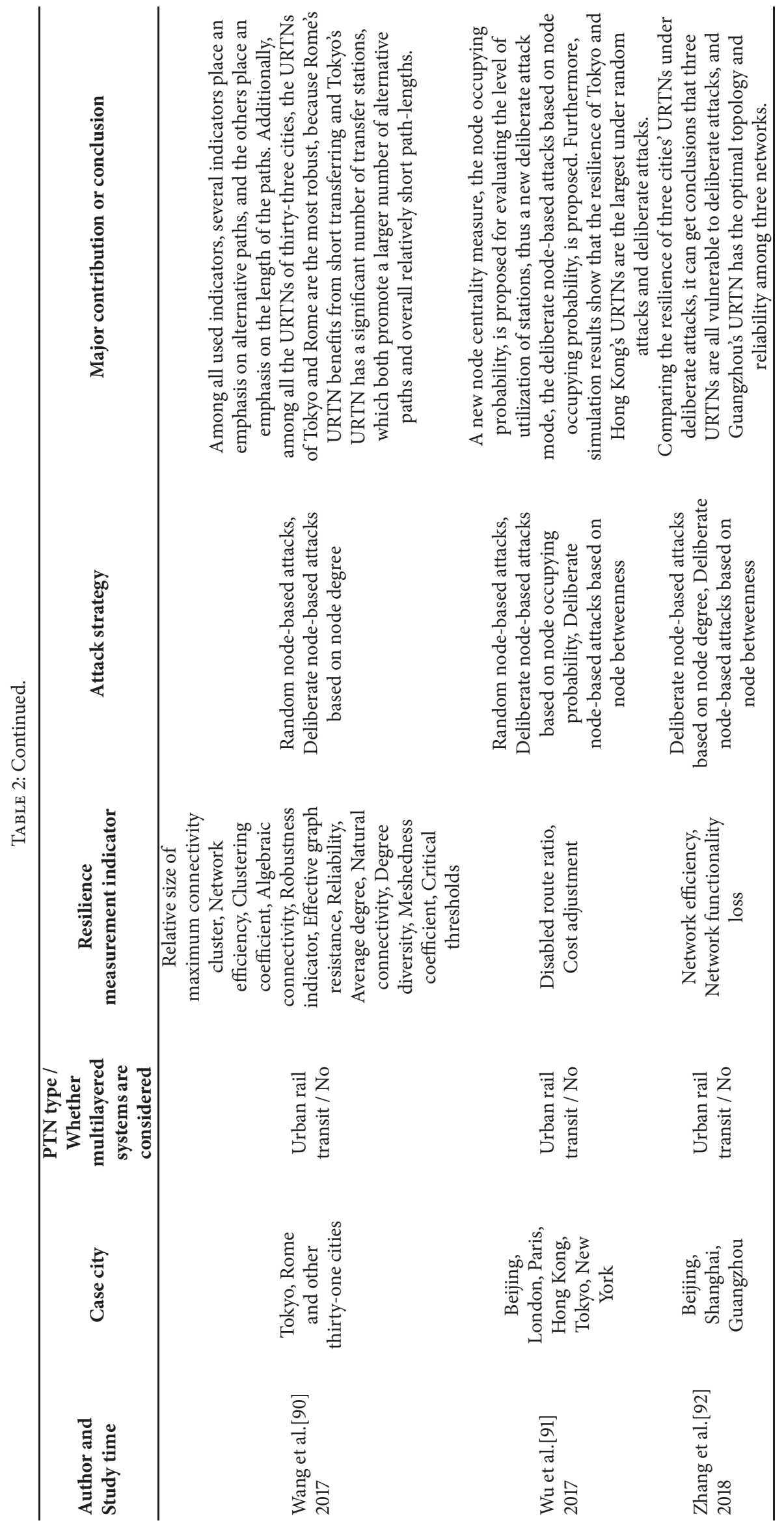


most of PTNs listed in Table 2 have this characteristic. This study area can be summarized as that, through applying the complex network approach, the static resilience of various cities' PTNs or the static resilience of various theoretical PTN models under various attack scenarios (in general, there are two categories: random attacks and deliberate attacks) is analyzed. It should be noted that, in this framework, there is no traffic flow or failure load spreading in the PTN, while it is committed to directly removing some nodes, edges, or even routes (see the Table 2, attack strategy) of network by simulating various attack scenarios, for observing the impact of these actions on the dynamic evolution of resilience criteria (various resilience measurement indicators). To sum up, some of the results and methods obtained from the aforementioned works may be useful for planning PTN or assessing the risk of PTN.

(b) The connections between the static resilience of PTN and the PTN complexity are so clear that the PTN complexity is discussed first in most studies on the static resilience of PTN. In other words, PTN complexity is the basis for understanding the static resilience of PTN.

(i) On the one hand, recent studies $[26,98]$ had shown how the network topological characteristics that are analyzed based on the complex network approach, offering an useful insight into identifying the most critical nodes (hubs) of transportation network. Such hubs with resilience conditions can then impact the resilience of entire network; e.g., Ferber et al. [82] had found that the network integrity is controlled by less than $0.5 \%$ of the stations; the number is 19 for Paris and 34 for London. The identifying of hubs based on node degree, node betweenness, node strength (see Table 1, complexity indicator), or a new node importance evaluation index that combines degree and betweenness (Yang et al. [22]) is conducted because of pioneer contributions from the PTN complexity related studies. As mentioned above, the hubs are so crucial than the studies on static resilience of PTN actually pay special attention to them. More specifically, it is related to the deliberate attacks. Here we list all kinds of attack scenarios in Table 2 (attack strategy), which can be categorized in two ways for identifying their differences: (i) random attacks and deliberate attacks, (ii) node-based attacks, edge-based attacks, and route-based attacks. However, the most common use in actual operation is a hybrid way, e.g., deliberate node-based attacks based on node degree. Furthermore, taking the deliberate nodebased attacks based on node degree as an example, we present the detailed attack process for knowing how the simulation experiment is triggered: from the original state, the largest degree node (or a certain proportion of the nodes with the first largest degree, the second largest degree, the third largest degree, ... .etc.) will be removed from PTN and all the topological characteristics of PTN will be recalculated after the attacks; then, the largest degree node (or a certain proportion of the nodes with the first largest degree, the second largest degree, the third largest degree, etc.) at this moment will be removed from PTN and all the topological characteristics of PTN will be recalculated after the attacks too; and the attacks continue.

(ii) On the other hand, the various resilience measurement indicators actually come from the complexity indicators defined by the studies of PTN complexity, such as the average shortest path length, clustering coefficient, network efficiency, and average degree. Here we continue discussing the other resilience measurement indicators. The relative size of maximum connectivity cluster is used in most of listed references (12/21), which certainly has the common function of all resilience measurement indicators, i.e., to signal the quantitative change in the network behavior, especially a serious network crash and to locate the value of concentration of removed nodes at which this change occurs. Moreover, some modified resilience measurement indicators are proposed for advancing the static resilience study framework, such as the connected origin-destination (OD) ratio (Sun et al. [85]), a composite indicator considering the impacts of weighted average path length, weighted global efficiency, the number of route service passengers, and relative disruption probability (Sun et al. [24]), which both embedded the actual passenger flow for concerning with the practical engineering application and being close to reality. Wang et al. [90] summarized a resilience measurement indicator set which includes 14 indicators widely used in network science and graph theory, and the Pearson correlation among these indicators is investigated for assessing the performance in capturing the static resilience of URTN. Additionally, the network connectivity entropy ( $\mathrm{Wu}$ et al. [83]) and the network structure entropy (Fu et al. [86]) are both used to measure the static resilience of PTN, which are certainly novel and interesting for this study area. For example, Fu et al. [86] declared that the betweenness importance based network structure entropy can reveal the interaction mechanism among static resilience of BTN, station heterogeneity, and network structure entropy. More specifically, the entropy is a thermodynamic concept and widely used to measure the disorder of a system; then it is introduced in the complex systems study, because it can describe the structure of a complex system [83]. The more uniform the energy distribution is, the greater the entropy achievements will be, otherwise, the smaller the entropy is. It should be noted that the current resilience measurement indicators of static resilience of PTN are all the global measurement indicators or macroscopic measurement indicators because of the limitations of static resilience study framework. However, the measurement indicators that consider the local changes should also be developed for providing a fuller understanding of the PTN resilience, which has been solved in the study of dynamic resilience of single layered PTN, such as the work of Zhang et al. [32]. 
(c) Analyzing the item of "PTN type/whether multilayered systems are considered" (see Table 2), it is similar to the development pattern of PTN complexity (see Section 2.2 (b)); i.e., the studies on static resilience of PTN have been developed from the static resilience of single type or single layer PTN into the static resilience of multitype or multilayer composite PTN, promoting the preliminary discovery of the interdependent relationship and the interaction among the subnetworks. More specifically, on the one hand, it focuses on the static resilience of one single type selected from BTN and URTN. Subsequently, it has developed to focus on the static resilience of composite PTN which is combined by two or more types of BTN, URTN, and public bicycle network (Bao et al. [25]). On the other hand, it focuses on the static resilience of one single layer selected from public transit geographical network layer, public transit transfer network layer, and public transit route network layer. Subsequently, it has developed to focus on the static resilience of composite PTN which is combined by two or more layers of public transit geographical network layer, public transit transfer network layer, and public transit route network layer (Ren et al. [89]).

In particular, it should be noted that an important advance has been made in this item. Bao et al. [25] gave a clear approach to couple station between bus station and urban rail transit station; i.e., a coupled method for bus station and urban rail transit station that considers the geographical proximity is proposed. This improvement will also advance the dynamic resilience of PTN, which will be discussed in Section 5, dynamic resilience of interdependent PTN.

(d) Other analyses for better capturing the main features of this study area are summarized below.

(i) The precise modeling for actual PTN promotes a new understanding of the static resilience of PTN, but it also further amplifies the defects of this study framework. More specifically, on the one hand, the actual PTN is just abstracted as a network model according to the Space L method, Space P method, or Space R method and directly subjected to static resilience simulation analysis, while it does not consider the existence of certain intrinsic connections between different spatial forms of empirical cities and the static resilience of PTN. Subsequently, based on the advances in PTN modeling methods and the consideration of various weights that have been mentioned in Section 2.2 (a), it has developed to focus on the special spatial characteristics such as the valley topography (Feng et al. [87]). This feature makes the network under deliberate attacks be more vulnerable than random attacks. On the other hand, this study area pays more attention to the static resilience of unweighted PTN and ignores some certain driving influences of network weights on the network topology. Subsequently, it has developed to focus on the static resilience of weighted PTN considering the influences of various weights (they have been mentioned in Section 2.2 (a)); e.g., Chen et al. [88] found that the resilience of weighted URTN is more obvious, and analyzing static resilience through the topology alone will overestimate the network reliability.

(ii) The static resilience study framework focuses on analyzing PTN reliability from the perspectives of macroscopic, static, topological, and theoretical aspects. It is essentially still at the level of network topology resilience and does not involve the complex dynamics evolution characteristics (e.g., the failure load (passenger flow) dynamic redistribution and the spreading characteristic), indicating that this study area is still far from the actual engineering application. In particular, Sun et al. [24] investigated passengers' distribution and redistribution across the URTN, which seems to advance the static resilience study framework in some extent. Actually, the socalled passenger redistribution is not essentially a dynamic passenger redistribution which exists in the dynamic resilience study framework, because the dynamic passenger redistribution is a dynamic and continuous evolution process, not just a one-time redistribution process that does not consider the impacts of redistribution passengers on other stations or routes. Nevertheless, the static resilience of PTN is the basis and prerequisite for analyzing the complex dynamic evolution mechanism of PTN resilience. Consequently, it is an indispensable step to study the dynamic resilience of PTN which is much closer to the actual PTN operation.

\section{Dynamic Resilience of Single Layered PTN}

4.1. Review. The dynamic resilience of urban PTN originates from the cascading failures phenomenon existing in complex network, and it is one of the important components of PTN reliability. An example of cascading failures among the real world networks is that three EHV transmission lines in Ohio of America malfunctioned in 2003, resulting in an accident at a power plant in this area. The frequency changes affected the whole network instantaneously, causing a cascading collapse effect. Finally, the cascading failures caused large-scale blackouts in eight US states and two Canadian provinces, affecting about 50 million residents, losing a load of about 6,1800 MV and an economic loss of about 30 billion US dollars. Study on the PTN complexity has proven that urban PTN is a kind of typical complex network in the real world, so the PTN is also facing the severe challenge of preventing cascading failures. To fully understand the study progress, this paper summarizes the studies on the dynamic resilience of single layered PTN from ten aspects: study time, case city, PTN type/whether multilayered systems are considered, station initial load definition, station capacity definition, station state, rule of failure load dynamic redistribution, resilience measurement indicator, attack strategy, and major contribution or conclusion, as shown in Table 3.

4.2. Analysis. Through reviewing the studies on dynamic resilience of single layered PTN, we can find that the study 


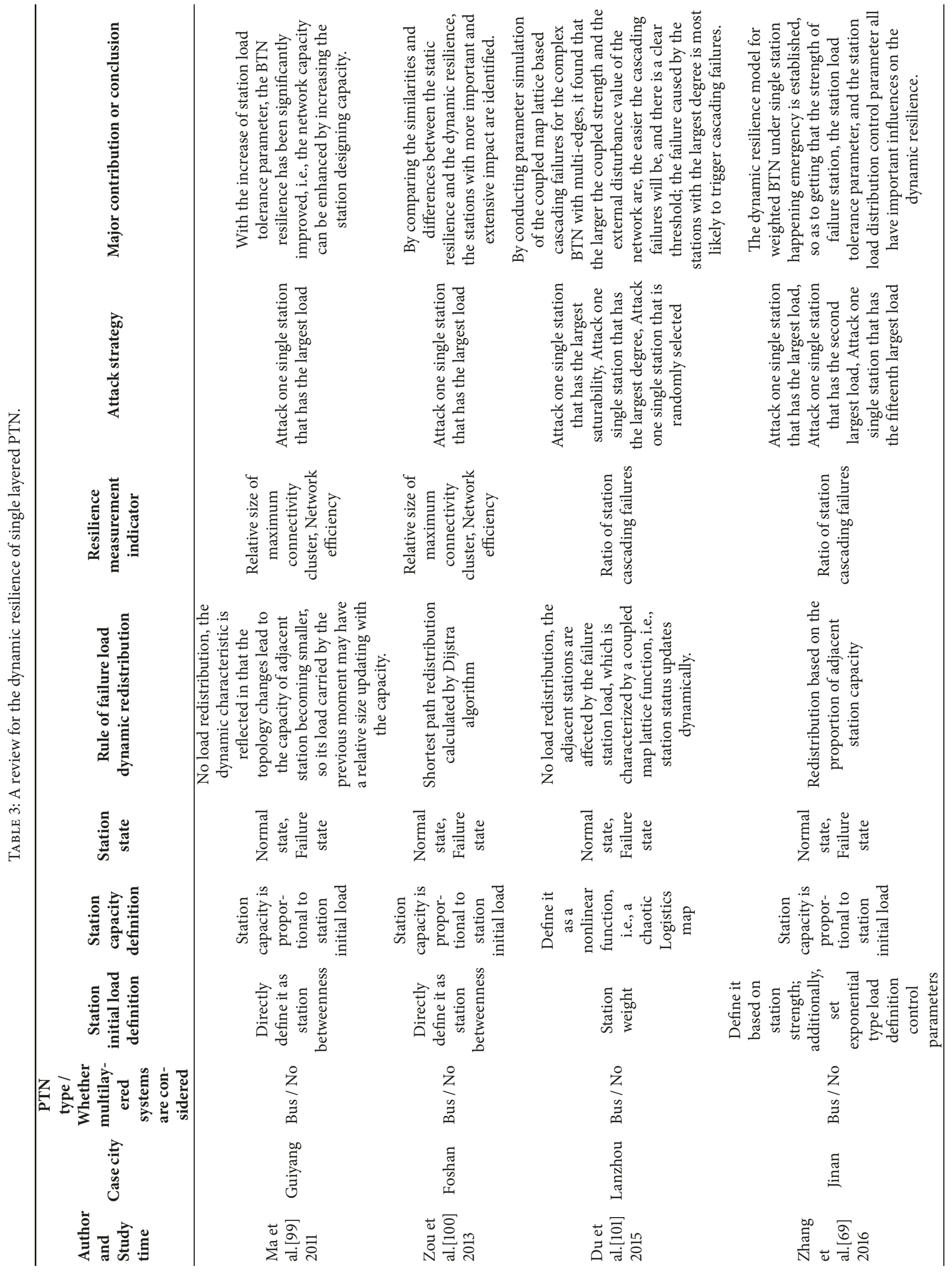




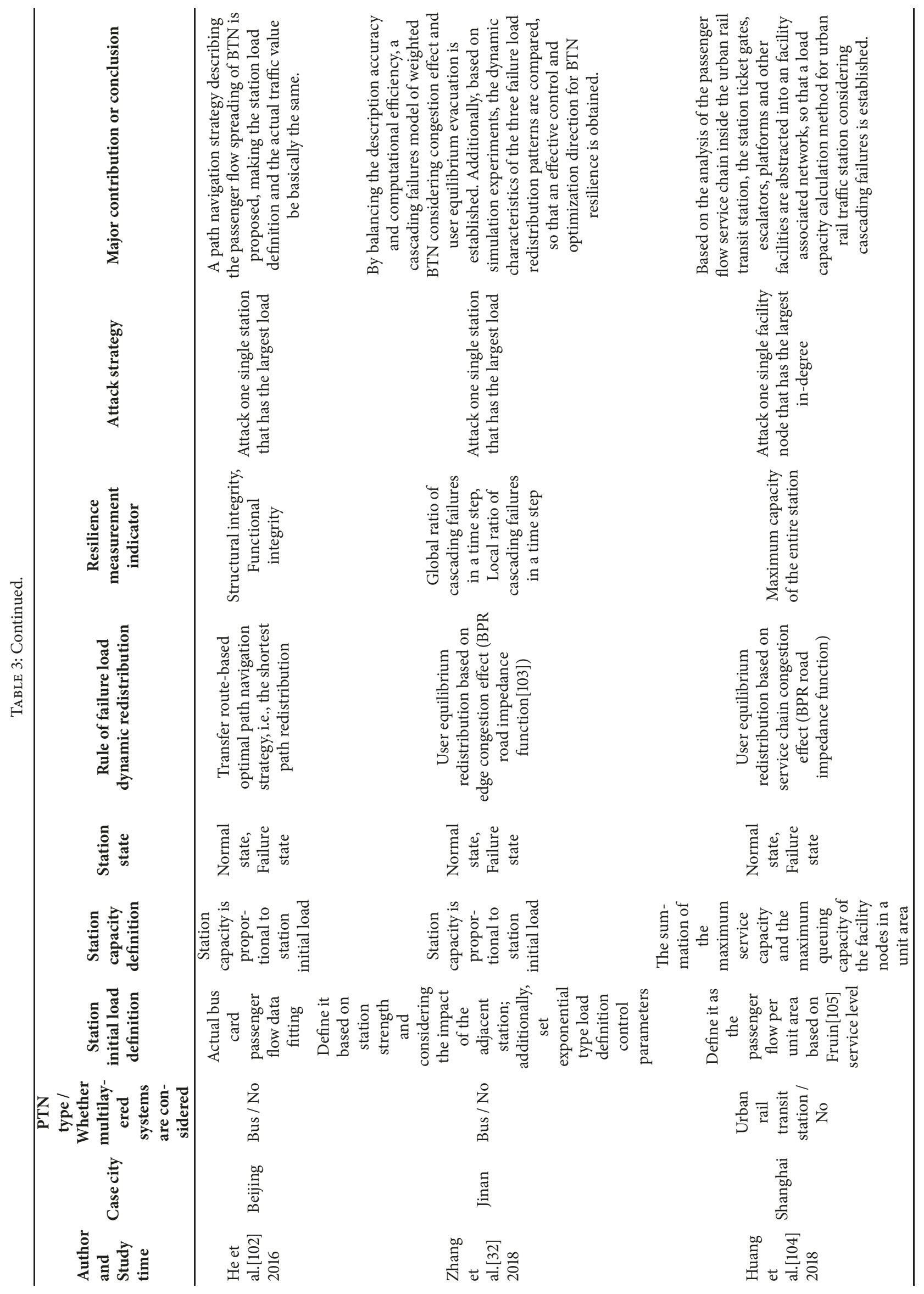




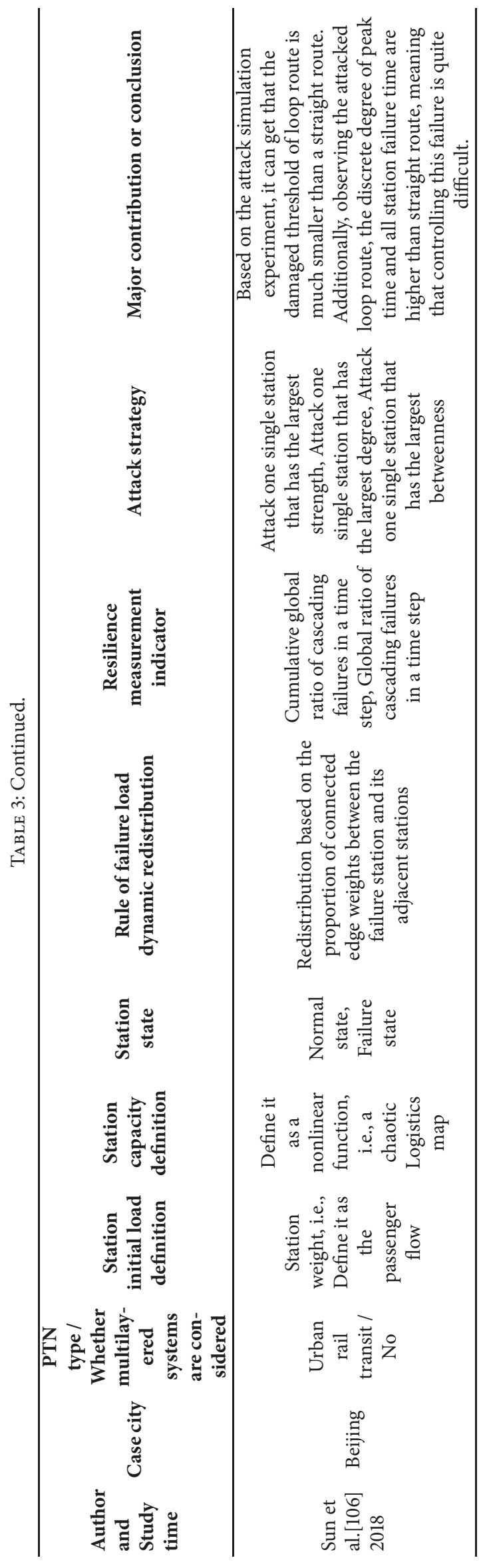


time is concentrated on 2011 to 2018, and the scholars have applied complex network theory to conduct extensive case studies on the dynamic resilience of single layered PTN, involving many cities. This study area is still a frontier area of complex network study, which presents the following features.

(a) The dynamics process involved in this study is relatively complicated. At present, much less scholars have studied its complex evolution mechanism. The related studies are summarized into two categories, the load-capacity model [107] and the coupled map lattice model [108], and the load-capacity model is used in most studies. Therefore, the dynamic resilience of single layered PTN based on the loadcapacity model can be described as that if an emergency occurs at a key station ((i) possibly from within the network, such as sudden large passenger flow accumulating at a station, exceeding its carrying capacity or (ii) possibly from outside of the network, such as urban traffic jams and terrorist attacks that cause the station to lose its normal function), the passenger flow carried by it will be evacuated to adjacent stations, which may cause passenger flow in adjacent stations to exceed their own capacity and continue to evacuate the passenger flow. This process sources gradually expand outwards, and it may eventually lead to the collapse of entire PTN. Obviously, the problems with urban PTN are clearly similar to those in power grids; e.g., the loadcapacity models for modeling cascading failures in power grids and urban PTN have no essential difference in the application framework. It should be noted that the cascading failures in power grids are more easily expanded outwards, because the electricity are redistributed to the adjacent power stations with unconscious behaviors. However, the similar redistribution process in urban PTN is driven by a conscious dynamic game process of the drivers and passengers of public transit vehicles. Thus, the commonalities and dissimilarities between urban PTN and power grids are the starting point for understanding the application of complex network approach in the real world network.

In particular, for better understanding the connections among various key items, we summarize a typical example showing the steps of numerical simulation analysis of dynamic resilience of single layered PTN (based on the loadcapacity model), shown as Table 4 .

An important missing part in step 2 (trigger the cascading failures) is actually ignored by most related studies or at least fails to give adequate explanations, i.e., the potential sources of disruption that generate the failure of the system are not clearly characterized, leading to a confusion that any potential disruption may cause the complete breakdown of nodes or edges. For overcoming this deficiency, Mattsson et al. [18] made some useful distinctions between internal and external causes of disruption and between accidental events and intentional interferences for transport system, thereby the potential sources of disruption include four categories: (i) accidental event caused by internal source, such as technical failures and mishaps; (ii) accidental event caused by external sources, such as adverse weather conditions and natural disasters; (iii) intentional interference by internal source, such as labor market conflicts; (iv) intentional interference by external source, such as pranks and antagonistic attacks. In a nutshell, the aforementioned category is more about disruption reason, while that in the static and dynamic resilience studies of PTN concerns more with the disruption consequence. In other word, the static and dynamic resilience studies of PTN only concern the disruption sources (actually, they may come from aforementioned sources categories (i), (ii), and (iv)) that can generate the node or edge failure. In the simulation, they are called the random attacks or deliberate attacks without characterizing potential sources of disruption. However, the various potential sources of disruption should be considered in the description of simulation attack strategy because of the following reasons.

(i) On the one hand, the current study framework thinks a node (edge) with two states, is either normal or failure in a time point, i.e., a binary performance. However, a node (edge) should have an intermediate state actually that is not noticed enough, called the crowded state (it is an abnormal state but not so serious as to be complete failure). Therefore, if the specific disruption source is not given, these three (or more) states cannot be well defined and distinguished, limiting the engineering applicability of this study framework.

(ii) On the other hand, if the specific disruption source is given in the simulation, the two types of cascading failures in PTN can be well defined and distinguished, i.e., the cascading failures under normal circumstance and the cascading failures under emergency. Under the former circumstance, only a proportion load of failure node (edge) is redistributed to the adjacent nodes (edges), the "failure" node (edge) can still carry the normal portion of the load. Under the latter circumstance, all the load of failure node (edge) is redistributed to its adjacent nodes (edges) and the failure node fails completely and cannot carry the load. In particular, the former one seems to occur more frequently in actual PTN operation, which can improve the engineering applicability of this study framework.

(b) This study area has been developed from the direct application of the theoretical model into the precise modeling that considers the characteristics of transportation engineering; i.e., we have the following.

(i) Initially, the station initial load is directly defined as the topological parameters such as the station betweenness (Ma et al. [99], Zou et al.[100]). Subsequently, it has developed to consider the impact of the weights (station strength) and the adjacent stations ( $\mathrm{Du}$ et al. [101]) or define it based on the actual bus card passenger flow data fitting ( $\mathrm{He}$ et al. [102]). Additionally, the exponential type load definition control parameters are set to control the distribution of network initial load (Zhang et al. [69], Zhang et al. [32]), or the station initial load is defined by determining the passenger flow per unit area based on Fruin [105] service level (Huang et al. [104]). 
TABLE 4: Steps of numerical simulation analysis of dynamic resilience of single layered PTN.

\begin{tabular}{|c|c|c|}
\hline Step number & Step name & Main operation \\
\hline 0 & Initialization & $\begin{array}{l}\text { Compute the initial load and capacity of all stations in the PTN (see the detailed } \\
\text { introduction in the Table 3, station initial load definition, and station capacity } \\
\text { definition) }\end{array}$ \\
\hline 1 & $\begin{array}{l}\text { Trigger the cascading } \\
\text { failures }\end{array}$ & $\begin{array}{l}\text { The cascading failures are triggered by attacking a single station that has a certain } \\
\text { characteristic (see the detailed introduction in the Table } 3 \text {, attack strategy). }\end{array}$ \\
\hline 2 & $\begin{array}{l}\text { Search adjacent } \\
\text { stations of the } \\
\text { attacked station }\end{array}$ & $\begin{array}{l}\text { There are some edges between the attacked station and its adjacent stations. These } \\
\text { edges will be used to transit the redistribution loads from the attacked station. }\end{array}$ \\
\hline 3 & Redistribute the load & $\begin{array}{l}\text { The redistribution loads from the attacked station are redistributed to the adjacent } \\
\text { stations according to certain rule (see the detailed introduction in the Table } 3 \text {, rule } \\
\text { of failure load dynamic redistribution). }\end{array}$ \\
\hline 4 & $\begin{array}{l}\text { Compare and } \\
\text { estimate the station } \\
\text { state }\end{array}$ & $\begin{array}{l}\text { Compare updated station load with station capacity (this operation is conducted to } \\
\text { all receiver stations of redistribution loads), if the updated station load is larger than } \\
\text { the station capacity, the station will be failed (failure state, see the detailed } \\
\text { introduction in the Table } 3 \text {, station state). Then, go on to carry on the step } 2 \text { and } \\
\text { step3 successively. Furthermore, carry on this process repeatedly, until no stations } \\
\text { occur cascading failures any more, and at this moment, the whole process of } \\
\text { cascading failures ends. }\end{array}$ \\
\hline 5 & $\begin{array}{l}\text { Compute the } \\
\text { resilience } \\
\text { measurement } \\
\text { indicators }\end{array}$ & $\begin{array}{c}\text { Compute various resilience measurement indicators before and after cascading } \\
\text { failures occurring. In particular, if resilience measurement indicator is a local } \\
\text { indicator or an indicator measuring each time step, this resilience measurement } \\
\text { indicator should be computed in every time step (a typical example can be seen in } \\
\text { the work of Zhang et al.[32]). }\end{array}$ \\
\hline 6 & $\begin{array}{l}\text { Change the value of } \\
\text { the control } \\
\text { parameters of } \\
\text { cascading failures } \\
\text { based dynamic } \\
\text { resilience model }\end{array}$ & $\begin{array}{l}\text { The cascading failures based dynamic resilience model of single layered PTN has } \\
\text { various control parameters for better controlling the model to be more realistic. } \\
\text { Thus, change the value of various control parameters and repeat the steps above, so } \\
\text { that a more complete and comprehensive numerical simulation analysis is } \\
\text { conducted (a typical example can be seen in the work of Zhang et al.[69]). }\end{array}$ \\
\hline
\end{tabular}

The precise considerations of the definition of station initial load also bring a significant improvement in the definition of station capacity, because the station capacity is proportional to station initial load in most works based on the general assumption of loadcapacity model [107] (see the detailed introduction in Table 3, station capacity definition). It should be noted that the station capacity defined by Huang et al. (the summation of the maximum service capacity, and the maximum queuing capacity of the facility nodes in a unit area) seems to provide a much better way that is close to the engineering application [104], the summation of the maximum service capacity, and the maximum queuing capacity of the facility nodes in a unit area seem to provide a much better way that is close to the engineering application. In terms of station state, the related studies are summarized into two states: the normal state and the failure state. More specifically, the state of a station can be obtained by comparing the relative size of updated station load and station capacity (see the detailed introduction in Table 4, step 4).

(ii) Initially, the rule of failure load dynamic redistribution involves only the dynamic update of station states and does not have the strict load redistribution process ( $\mathrm{Ma}$ et al. [99]); or the spreading process involves the failure load redistribution based on the proportion of adjacent station capacity (Zhang et al. [69]), the shortest path redistribution (Zou et al. [100], He et al. [102]), but does not consider the congestion effect on edge (road section) and the conscious dynamic game process existing in path selection behavior. Subsequently, it has developed to introduce the congestion effect of road section based on the BPR road impedance function[103], so that the dynamic resilience model of single layered PTN based on user equilibrium evacuation is further established (Zhang et al. [32], Huang et al. [104]).

(c) The study scale and measurement indicator scale of this study area have been further expanded to significantly complement and improve this study framework; i.e., we have the following.

(i) In terms of the study scale expanded, it initially focuses on the failure load redistribution process outside the station (redistributing load among multiple stations); i.e., the traditional resilience study framework of PTN that is a cascading failures based mesoscopic resilience model. Subsequently, it has developed to focus on the failure load redistribution process inside a large hub transfer station (redistributing load inside a single station), i.e., a cascading 
failures based microcosmic resilience model (Huang et al. [104]). The ticket gates, escalators, platforms, and other facilities in a single urban rail transit station can be abstracted into a facility associated network; thus a cascading failures based resilience model for this network can be established. In particular, this operation can be associated with the attack strategy that triggers the cascading failures of the PTN (e.g., attack one single station with the largest load); thus a more complete failure load redistribution process is considered; i.e., a failure originates from a certain facility inside a urban rail transit station; the failure load will be redistributed in the facility associated network; then it expands to the outside of this station through the exit facility. At this moment, this operation is equivalent to triggering the cascading failures of the PTN through the attack strategy mentioned in Table 3, attack strategy.

(ii) In terms of the measurement indicator scale expanded, some global resilience measurement indicators, which are commonly used in the study of static resilience of PTN, are also used to measure the dynamic resilience of single layered PTN (e.g., the relative size of maximum connectivity cluster and the network efficiency used in the works of Ma et al. [99], Zou et al. [100]). Then, a novel indicator, the ratio of station cascading failures that concerns how many stations are failed because of the cascading failures, is proposed for providing a novel way to capture the failure process. However, it is still a global resilience measurement indicator that pays more attentions to representing the dynamic evolution of entire network. Recently, this study area has developed to focus on the local strength of cascading failures at each time step (corresponding to a set of failure load redistribution process that happened at the same time, maybe between multiple failed stations and their adjacent stations), i.e., the local ratio of cascading failures in a time step (Zhang et al. [32]). Due to the proposed time step concept, the dynamic resilience measurement indicators have developed from 2 dimensions into 3 dimensions; the 3 dimensions indicators can intuitively describe the complex dynamic evolution process of cascading failures. Additionally, another measurement indicator proposed in the same work [32], the global ratio of cascading failures in a time step, is a modified trying for the ratio of station cascading failures through recording the station cascading failures in each time step.

(d) The continuous improvement process presented in the aforementioned literature is essentially a process that gradually makes the study framework be closer to the actual engineering application, e.g., the scholars with a background of transportation engineering always hope to identify a possible method to quantitatively control the dynamic resilience of single layered PTN, so that this interdisciplinary study area can be further expanded. Recently, a work of Zhang et al. [32] tried to contribute to this problem. More specifically, they compared the different dynamic characteristics of three failure load redistribution patterns to obtain a clear implementation measure for effectively controlling the dynamic resilience of PTN. As a result, the control optimization for the dynamic resilience of PTN is no longer limited in the stage that only provides the qualitative optimization countermeasure; i.e., it is an effective try to quantitatively control the dynamic resilience of single layered PTN.

Additionally, most related works always claim that the cascading failures of PTN are triggered by attacking one single station with the largest load; however, there is no further works to study how the cascading failures occur inside a large integrated passenger station. The large integrated passenger station is a special node in the PTN, and it is usually formed by the intersection of several modes of public transit, such as the Nanjingnan station (located in Nanjing city, China) formed by the intersection of bus, urban rail transit, rapid transit railway, and taxi. It should be noted that the various facilities (station ticket gates, escalators, platforms, etc.) passed by passengers in a large integrated passenger station can be abstracted as a facility associated network, thus the cascading failures maybe occur on some facility nodes. Recently, a work of Huang et al. [104] focused on this phenomenon. More specifically, they studied the dynamic resilience of an facility associated network inside a urban rail transit station based on the cascading failures perspective, applying this study framework to a more microscopic and detailed engineering problem; i.e., a load capacity calculation method of urban rail transit station considering cascading failures perspective is developed based on the describing method for initial load and capacity of facility nodes.

\section{Dynamic Resilience of Interdependent PTN}

5.1. Review. With the new development of complex network theory, the scholars have realized that many real world networks do not exist in isolation, but consist of multiple subnetworks with interdependent relationship, i.e., the interdependent networks [17, 109-111]. An interdependent network is generally composed of two or more layers such as an infrastructure connection network and an operation association network. The interdependent relationship among the subnetworks makes the failure of any subnetwork potentially affect its interdependent network and further feedback to the failure source network through the interaction among subnetworks, i.e., the cascading failures of an interdependent network [112116]. An example among the real world networks is that, in 2015, the power grids in various regions of Ukraine were attacked by hackers, the control servers of the underlying generators or substations were shut down, and the sensing and control functions of the corresponding physical devices were lost, resulting in the operational disruptions of some devices. More seriously, the virus spread widely through information network, and it led to the grid company losing contact with multiple power plants and substations, which further lost the sense and real-time control of power grid equipment. Ultimately, these interactions caused the grid company to fail to make correct decisions and schedules, leading to widespread blackouts. The BTN is not isolated operation in 
the urban transportation system but has an interdependent relationship with the road network or URTN; thus there is corresponding cascading failures problem of interdependent PTN. Considering the engineering science background, it can also be called the dynamic resilience problem of multimodes public transit system. To fully understand the study progress, this paper summarizes the studies on the dynamic resilience of interdependent PTN from twelve aspects: study time, case city, PTN type/whether multilayered systems are considered, station initial load definition, station capacity definition, station state, rule of failure load dynamic redistribution, type of interdependent network, interdependent type between interdependent stations, resilience measurement indicator, attack strategy, and major contribution, or conclusion, as shown in Table 5.

5.2. Analysis. Through reviewing the studies on dynamic resilience of interdependent PTN, we can find that the study time is concentrated on 2014 to 2018, and the scholars have applied complex network theory to conduct extensive exploratory studies on the dynamic resilience of interdependent PTN, involving many cities. This study area is the frontier study area of complex networks that has emerged in recent years, which presents the following features.

(a) The aforementioned studies are based on the work of Buldyrev et al. [17]. They are essentially the cascading failures model based on topological structure, and they abstract urban PTN as an interdependent network and systematically reveals the complex evolution mechanism of cascading failures in the interdependent PTN. However, most of the studies focus on constructing effective models that describe the resilience evolution. Additionally, the studies on the control and optimization of resilience mostly focus on the formulation of qualitative optimization strategies but do not achieve quantitative optimization control with real-time, dynamic, and interactive evolution. It should be noted that there is a lack of extension study directions that can promote the engineering application of the resilience modeling technology for complex PTN. In other words, this study area is still far from the engineering application.

In particular, for better understanding the connections among various key items, we summarize a typical example showing the steps of numerical simulation analysis of dynamic resilience of interdependent PTN (based on the load-capacity model) shown as Table 6 . It is similar to that of single layered PTN, and the main differences are in step 2 and step 3 due to considering the existence of interdependent stations of the attacked station.

(b) The modeling methods of interdependent PTN have been discussed in Section 2.2 (b), which are essentially the combination of six network modeling methods. The various types of interdependent network are divided into two categories, i.e., the coupled network constructed by different types of PTN and the coupled network constructed by different represented layers of a single type of PTN (see the detailed introduction in Section 2.2 (b)). In particular, different from the multilayered systems studied in Section 2 (Latora et al. [34], Ferber et al. [37], Sienkiewicz et al. [38], Ferber et al. [40], Ferber et al. [47], Berche et al. [48], etc.), the multilayered systems in this section are all the strict ones, because these interdependent networks are established based on the clear definition of interdependent type between interdependent stations (see the detailed introduction in Table 5, interdependent type between interdependent stations). It should be noted that although no clear coupled method for clarifying the interdependent type between interdependent stations is declared in the work of Huang et al. [73], the differences between bus route and urban rail transit route are virtually considered based on weighting the actual passenger flow (it is recorded by the IC system) on the routes. Thus, the interdependent PTN in the work of Huang et al. [73] is also a strict one.

(c) The strategies to improve the PTN resilience under complex network framework are inadequate, so this issue is not sufficiently rich to be listed in the aforementioned overview tables. In most static resilience studies of PTN, the obtained improvement strategy is only the qualitative optimization strategy not a quantitative control method, such as doing the best effort to protect the key nodes (edges) with largest degree, strength, or betweenness. This strategy is proposed because such key nodes (edges) can make the PTN be vulnerable, if they are removed in the deliberate attacks simulation. In most dynamic resilience studies of PTN, the obtained improvement strategy also includes the qualitative optimization strategy, such as doing the best effort to protect the key nodes (edges) with largest load or increasing the network redundancy by increasing the load tolerance parameter that is used to control how many load a node can carry. Moreover, the control directions (increase or decrease) of various control parameters that are used for better controlling the cascading failures model to be more realistic are given as the preliminary quantitative control method by numerical simulation analysis (a relatively comprehensive control parameters numerical simulation can be seen in the work of Zhang et al.[71]).

However, the aforementioned strategies are essentially not the strategies with engineering applicability (i.e., they are not the strict quantitative control methods), because the current PTN resilience study framework focuses on establishing an effective model to describe the PTN resilience. Therefore, the quantitative control method with engineering applicability for improving the PTN resilience is really needs to be developed; e.g., a preliminary study has been conducted by Zhang et al. [32]; they found that guiding the transforming among varying failure load dynamic redistribution patterns (FLDR pattern following average evacuation[121], FLDR pattern based on the proportion of adjacent node capacity $[69,122]$, and FLDR pattern following user equilibrium evacuation [32]) through some technical measures or traffic policies, may be the control direction that can make efforts for improving the PTN resilience.

(d) The dynamic resilience of interdependent PTN is an exploratory study. This study area focuses on the perspectives of network physical topology and the system science, while it does not consider the user selection behavior and transportation engineering characteristics. If the engineering application of this study area is wanted to advance, the following defects are needed to solve the following. 


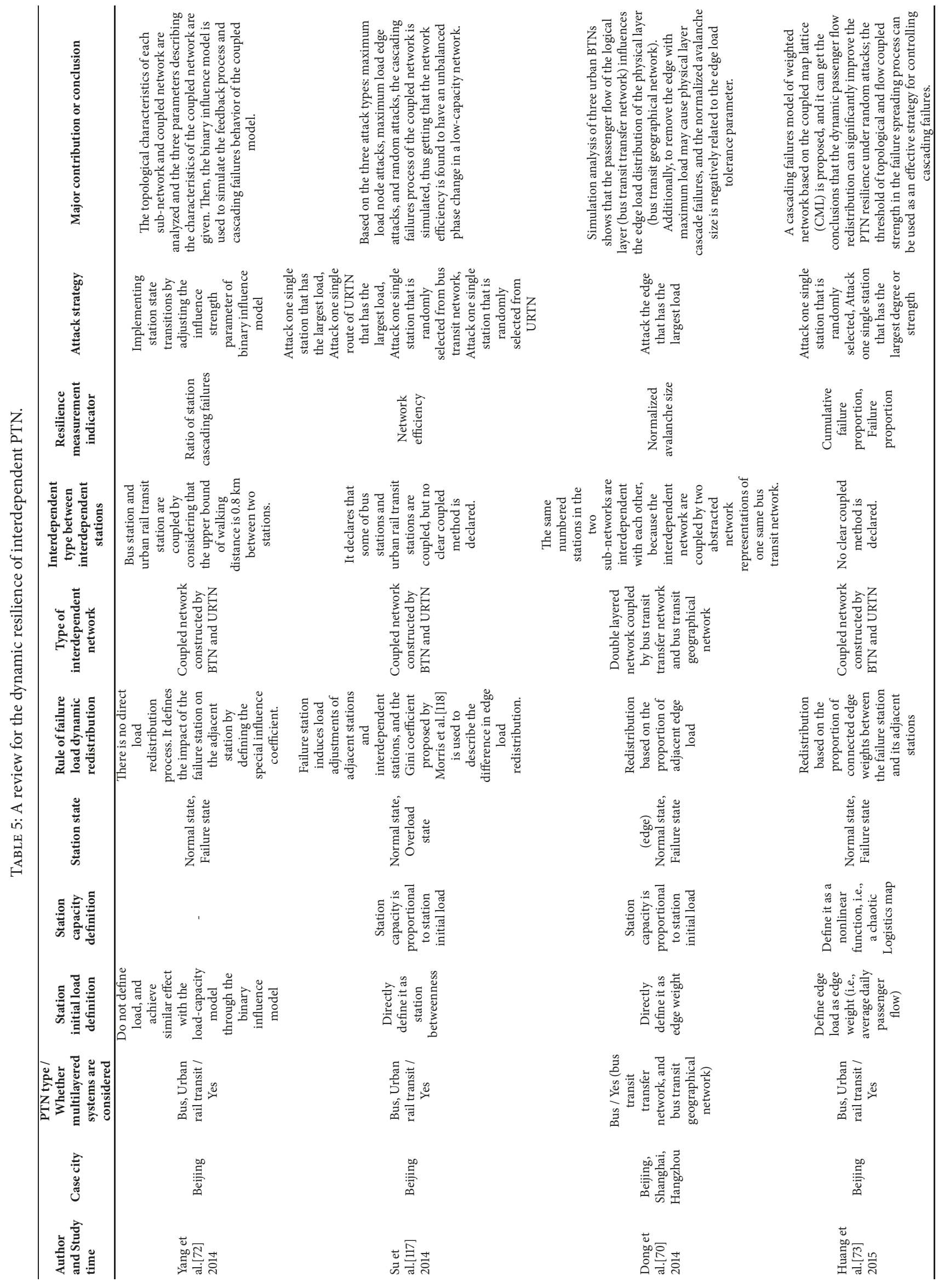




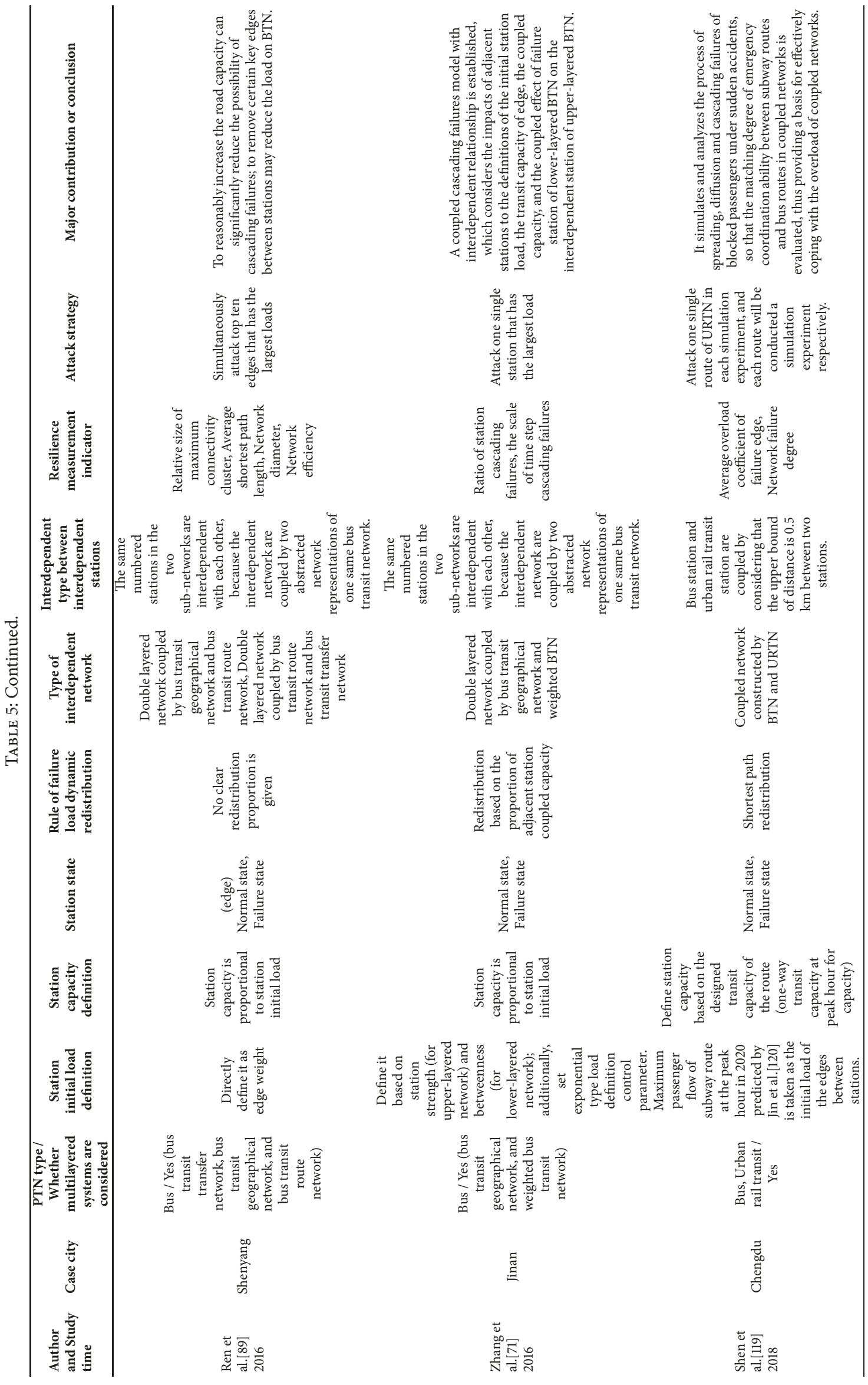


TABLE 6: Steps of numerical simulation analysis of dynamic resilience of interdependent PTN.

\begin{tabular}{|c|c|c|}
\hline Step number & Step name & Main operation \\
\hline 0 & Initialization & $\begin{array}{l}\text { Compute the initial load and capacity of all stations in the interdependent PTN (see } \\
\text { the detailed introduction in the Table 5, station initial load definition, and station } \\
\text { capacity definition) }\end{array}$ \\
\hline 1 & Trigger the cascading failures & $\begin{array}{l}\text { The cascading failures are triggered by attacking a single station that has a certain } \\
\text { characteristic (see the detailed introduction in the Table 5, attack strategy). }\end{array}$ \\
\hline 2 & $\begin{array}{l}\text { Search adjacent stations and } \\
\text { interdependent stations of the } \\
\text { attacked stations }\end{array}$ & $\begin{array}{l}\text { On the one hand, there are some edges between the attacked station and its adjacent } \\
\text { stations. These edges will be used to transit the redistribution loads from the } \\
\text { attacked station. On the other hand, the interdependent station of the attacked } \\
\text { station is also influenced by the failure of the attacked station. }\end{array}$ \\
\hline 3 & $\begin{array}{l}\text { Redistribute the load to the } \\
\text { adjacent stations and impose } \\
\text { coupled effect to the } \\
\text { interdependent stations }\end{array}$ & $\begin{array}{l}\text { For the adjacent stations, the redistribution loads from the attacked station are } \\
\text { redistributed to the adjacent stations according to certain rule (see the detailed } \\
\text { introduction in the Table 5, rule of failure load dynamic redistribution). } \\
\text { For the interdependent stations, the coupled effect is imposed by considering the } \\
\text { combined influences of the type of interdependent network and the interdependent } \\
\text { type between interdependent stations (see the detailed introduction in the Table 5, } \\
\text { type of interdependent network, and interdependent type between interdependent } \\
\text { stations). }\end{array}$ \\
\hline 4 & $\begin{array}{l}\text { Compare and estimate the } \\
\text { station state }\end{array}$ & $\begin{array}{l}\text { Compare updated station load with station capacity (this operation is conducted to } \\
\text { all receiver stations of redistribution loads and all influenced stations by coupled } \\
\text { effect), if the updated station load is larger than the station capacity, the station will } \\
\text { be failed (failure state, see the detailed introduction in the Table } 5 \text {, station state). } \\
\text { Then, go on to carry on the step } 2 \text { and step } 3 \text { successively. Furthermore, carry on this } \\
\text { process repeatedly, until no stations occur cascading failures any more, and at this } \\
\text { moment, the whole process of cascading failures ends. }\end{array}$ \\
\hline 5 & $\begin{array}{l}\text { Compute the resilience } \\
\text { measurement indicators }\end{array}$ & $\begin{array}{l}\text { Compute various resilience measurement indicators before and after cascading } \\
\text { failures occurring (see the detailed introduction in the Table } 5 \text {, resilience } \\
\text { measurement indicator). In particular, if resilience measurement indicator is a local } \\
\text { indicator or an indicator measuring each time step, this resilience measurement } \\
\text { indicator should be computed in every time step (a typical example can be seen in } \\
\text { the work of Zhang et al.[32]). }\end{array}$ \\
\hline 6 & $\begin{array}{l}\text { Change the value of the control } \\
\text { parameters of cascading failures } \\
\text { based dynamic resilience model }\end{array}$ & $\begin{array}{l}\text { The cascading failures based dynamic resilience model of interdependent PTN has } \\
\text { various control parameters for better controlling the model to be more realistic. } \\
\text { Thus, change the value of various control parameters and repeat the steps above, so } \\
\text { that a more complete and comprehensive numerical simulation analysis is } \\
\text { conducted (a typical example can be seen in the work of Zhang et al.[71]). }\end{array}$ \\
\hline
\end{tabular}

(i) In terms of the station capacity definition, it is oversimplified because not all stations' capacities are directly proportional to their initial loads; e.g., a common phenomenon existing in the actual PTN operation is that the station current passenger flow has actually exceeded its bearing capacity due to the lag of the station infrastructure upgrade. Thus, the initial load of this station based on passenger flow weighting has actually exceeded its bearing capacity. Similarly, the capacity of this station obtained by the load-capacity model (station capacity is proportional to station initial load) has been exaggerated in essence, resulting in the cascading failures model of interdependent PTN divorced from reality.

(ii) In terms of the station state, it is currently divided into the normal state and the failure state (overload state). But being significantly different from other complex networks, the drivers and passengers of public transit vehicles have subjective initiative and self-organization mobility. Thus, as long as the station passenger flow exceeds the design bearing capacity of station within certain limits, the station can gradually return to the normal state through self-organization capability; i.e., the station should also have an intermediate state, called the crowded state.

(iii) In terms of the rule of failure load dynamic redistribution, it does not conform to the actual operating characteristics of PTN. In particular, it does not consider the characteristics of transportation engineering and simply takes the failure load dynamic redistribution based on proportion of adjacent edge (or station) weights, or based on its variant or improved types. Such rules are simple and easy to operate, but it lacks the descriptions of the path selection behavior of drivers and passengers (users) and the congestion effect of road section when focuses on the engineering science background. Thus, it is difficult to avoid the large error and leads to be far from the engineering application. 
(iv) In terms of the resilience measurement indicator, it is mainly the topological parameters of complex network that characterize the aggregated behavior and the macroscopic topology, and it is the measurement indicators with single measurement function. In particular, it lacks the consideration of multiple perspectives such as the global and local perspectives and the aggregated and discretized perspectives, i.e., a multiperspective measurement indicator system that can intuitively and comprehensively measure the resilience. As a result, the recognition of the dynamic resilience of interdependent PTN is still at the stage of qualitative analysis.

\section{Summary and Prospect}

Complex network is an effective tool that can be used to analyze multidisciplinary problems, and it provides important ideas for the studies on the complexity and resilience of urban PTN. Following the development trend of complex network theory, the scholars have extensively and deeply studied the complexity and resilience of urban PTN from four aspects: the PTN complexity, the static resilience of PTN, the dynamic resilience of single layered PTN, and the dynamic resilience of interdependent PTN. Based on the perspective of interdisciplinary study, this paper abstracts several key items for summarizing the relevant literature, so that the basic study rules and key features of this interdisciplinary study area can be obtained.

The study on the PTN complexity is the basic work for the extension study of complex PTN such as the resilience of PTN, and it can be regarded as a preliminary study and an introduction to understand why the PTN is complex. The static resilience of PTN is a simplified treatment to understand the PTN resilience. It mainly considers the network resilience from the perspective of topological structure, making the PTN resilience be indistinguishable from other complex networks. The dynamic resilience of single layered PTN is an extension of static resilience study, considering the effect of a failure station on its adjacent stations. In particular, most of them are based on the load-capacity model, developing from the load distribution simply determined by topological structure into become into considering the weights influence based on topological structure. Additionally, Zhang et al. [32] and Huang et al. [104] both considered the congestion effect and thought that the failure load dynamic redistribution should follow user equilibrium rule, making the study framework be close to reality and transportation engineering characteristics in a large extent. However, with the increasingly close interdependent relationship of various networks, the limitation of the dynamic resilience of single layered PTN has become increasingly prominent. Thus, the dynamic resilience of PTN should be mapped to an interdependent network, so as to better reflect the evolutionary behavior mechanism of the actual network dynamic resilience. Currently, some exploratory studies on the dynamic resilience of interdependent PTN have been conducted, laying the basic framework for this study area. Moreover, to enhance the applicability of this framework, the "universality" of complex networks should be broken through; the "particularity" of PTN should be paid more attentions, e.g., the dynamic game behavior which exists in the evacuation route selection by passengers and drivers under emergencies should be considered. This game process involving human behavior will dramatically make the failure load dynamic redistribution be different from other complex networks.

Transportation engineering is a subject with highly application characteristic, so the application of the complex network theory on it should be close to the actual PTN operation. Additionally, it should not only be limited to the misunderstanding on considering the topological structure study as the supremacy, so that the study can truly guide the government department and public transit company to improve the efficiency of public transit management and to significantly improve the PTN reliability. Thus, in order to realize the development trend of cyclic and forward-complex network theory, network resilience theory, transforming into a realistic model and method that is close to actual public transit operation, engineering application and practice, and contributing to complex network theory, the following problems of this interdisciplinary study area are worth further study, i.e., developing the complex network theory related complexity and resilience study of urban PTN based on two purposes: enriching the complex network analytical framework and improving engineering applicability.

(a) Combining complex network theory and data mining when analyzing the complexity and resilience of PTN. As a novel trend in the complex network approach related studies, combining complex network theory and data mining has been prospected in the work of Zanin et al. [123]. This trend gradually forms based on such understandings; i.e., the data mining and complex network theory have the common objective to find feasible ways to represent various complex systems and ultimately provide a better understanding of their structure and dynamics. Thus, the same trend should be considered in the urban PTN. It should be noted that the difficulty of data acquisition in the PTN is much less than that in other complex networks because of the advanced public transit operation information management system based on GPS and the urban transportation infrastructure distribution information system based on GIS. Nevertheless, acquiring the data for study purposes at high level of detail is still a challenge because of the concerning on privacy, anonymity and business modes. This challenge should be given special attention because a complete public transit trip chain usually includes multiple public transit modes, while it limits access to the complete trip chain. Some Chinese cities like Beijing have begun constructing the integrated traffic data center for optimizing urban governance, and the use of data for the purposes of academic study is welcome after signing the nondisclosure agreement. This advanced trend should be further promoted for establishing a data verification environment platform that has high level of data detail and can be used for verifying various proposed traffic models.

Therefore, the real difficulty is the data mining that can help to establish a PTN model being closer to the reality; e.g., many scholars have reached a consensus; i.e., embedding the spatial coordinates, departure frequency of public transit, 
and actual passenger flow can help it a lot. In the future, the studies on complexity and resilience of urban public transit network should consider these embedding weights based on the novel method combining complex network theory and data mining, so that the obtained study results can be used in the engineering project to a large extent.

(b) Optimizing the general analytical frameworks of PTN complexity, static resilience of PTN, dynamic resilience of single layered PTN, and dynamic resilience of interdependent PTN. Although general analytical frameworks of four topics have been summarized in Section 2.2 (c), Section 3.2 (a), Section 4.2 (a), and Section 5.2 (a), they inevitably have many defects; e.g., we have the following.

(i) Identification of Space L, Space P, and Space R methods is a start only, but not sufficient in the study of PTN complexity. Once we have a much better network modeling method, the complexity indicator extracted from the established PTN model can reflect the real complexity of PTN. Furthermore, how can we possess a complexity indicator that is closer to the characteristics of actual PTN operation? The answer may come from establishing a connection between complexity study and transportation engineering technology. More specifically, as mentioned in our analysis section, the "harness" (Ferber et al. [40], Ferber et al. [47], Berche et al. [48], etc.) provides an example for using the complexity indicators to better understand the complex PTN in a more realistic way, and an example that the network with weaker "harness" characteristic maybe more resilient. In particular, it has a certain connection with a similar concept in urban bus transit planning project, i.e., the repetition coefficient of route. It should be noted that traffic engineers always try their best to reduce the repetition coefficient of bus route in actual bus transit planning practice. Maybe this connection is the bridge between the complex network theory and transportation engineering technology. Therefore, in the future, a wider application and validation of "harness" should be conducted on the PTN of various cities, as well as the certain connections between the "harness" and the repetition coefficient of route. Additionally, some other complexity indicators that are similar to the proposed way for "harness", as well as the complexity indicators comprehensively considering multilayered systems, should also be developed.

(ii) The credibility of various resilience measurement indicators needs to be tested in the future. The key issue is building the connection between the resilience measurement indicators and actual passenger evacuation behavior of PTN. However, this connection is difficult to establish just based on the simulation experiment with a lot of assumptions. Additionally, the actual passenger evacuation case of PTN is rare, because its detailed process is quite difficult to record and capture. Thus, the credibility verification method for various resilience measurement indicators should be developed through some ingenious thoughts, so that the analytical framework of complex network theory related study can be more complete. There is no doubt that it will be a very challenging work. Furthermore, it also should be noted that the other key terms (components) of PTN resilience model are all needed to modify through considering the engineering applicability, e.g., as the most basic component in the analytical framework of PTN resilience; the current definitions for station initial load are mostly imprecise, so a more precise estimation method for determining the station initial load should be developed in the near future.

(c) Developing the study directions that can promote engineering application. As mentioned in Section 5.2 (d), if the engineering application of dynamic resilience of interdependent PTN is wanted to advance, the four exampled defects need to be solved, so that the dynamic resilience of interdependent PTN is more precise and closer to actual PTN operations; e.g., it should give a reasonable estimate of station capacity based on factors such as the station space size and the characteristics of passenger flow organization. Additionally, it should consider the combined effects of the congestion effect on road section and the failure load dynamic redistribution following with various user equilibrium game mechanisms. Thus, a quantitatively controllable mesoscopic reliability model of an interdependent PTN, as well as its algorithms and evaluation grading technology can be established.

After having a reliable model describing the dynamic resilience of multimode PTN, how public transit routes should be laid when the PTN resilience is optimal can be inferred (i.e., the optimal route layout estimation technology based on reliability optimization), as well as developing the quantitative control method with engineering applicability for improving the PTN resilience, and clearly characterizing the potential sources of disruption that generate the failure of PTN. For the traditional traffic engineers, it is not enough to just know about the complexity and resilience of PTN, while they want to know the engineering applicability possessed by a novel analytical framework. In particular, these novel study directions can answer the questions asked by traditional traffic engineers well: what are the purposes and significances of applying complex network theory in transportation study area?

(d) Focusing on the cascading failures of PTN at much smaller scales, and modeling cascading failures based passenger evacuation inside and outside one key station within a unified study framework. In the current study framework, the cascading failures of PTN are triggered by attacking one single station with the largest load, but there is no further works to study how the cascading failures occur inside a large integrated passenger station. The large integrated passenger station is a special node in the PTN, and it is usually formed by the intersection of several modes of public transit, such as the Nanjingnan station (located in Nanjing city, China) formed by the intersection of bus, urban rail transit, rapid transit railway, taxi, etc., or the Hongqiao integrated transportation station (located in Shanghai city, 
China) that even includes air transportation. It should be noted that the various facilities (station ticket gates, escalators, platforms, etc.) passed by passengers in a large integrated passenger station can be abstracted as a facility associated network; thus the cascading failures maybe occur on some facility nodes. The cascading failures inside and outside the large integrated passenger station (i.e., the cascading failures of the facility associated network and the cascading failures of the interdependent PTN) can be connected through the exit facility nodes of the facility associated network that connect different modes of PTN. As a result, the cascading failures will be recognized more comprehensively in urban integrated transportation systems.

\section{Data Availability}

No data were used to support this study.

\section{Conflicts of Interest}

The authors declare that there are no conflicts of interest regarding the publication of this paper.

\section{Acknowledgments}

This work was supported by the National Natural Science Foundation of China (no. 51478110, no. 71471104, and no. 71871130), the Scientific Study Foundation of Graduate School of Southeast University (no. YBPY1884), the Postgraduate Study \& Practice Innovation Program of Jiangsu Province (no. KYCX17_0144), the Fundamental Study Funds for the Central Universities (no. KYCX17_0144), and the Science and Technology Program of Jiangsu Province (no. BY201607605).

\section{References}

[1] R. Cervero, "The Transit Metropolis : A Global Inquiry," Development, 1998.

[2] M. Estrada, J. Mensión, J. M. Aymamí, and L. Torres, "Bus control strategies in corridors with signalized intersections," Transportation Research Part C: Emerging Technologies, vol. 71, pp. 500-520, 2016.

[3] S.-X. He, "An anti-bunching strategy to improve bus schedule and headway reliability by making use of the available accurate information," Computers \& Industrial Engineering, vol. 85, article no. 3979, pp. 17-32, 2015.

[4] A. Fonzone, J.-D. Schmöcker, and R. Liu, "A model of bus bunching under reliability-based passenger arrival patterns," Transportation Research Part C: Emerging Technologies, vol. 59, pp. 164-182, 2015.

[5] S. Chakrabarti and G. Giuliano, "Does service reliability determine transit patronage? Insights from the Los Angeles Metro bus system," Transport Policy, vol. 42, pp. 12-20, 2015.

[6] J. W. Duncan and H. S. Steven, "Collective dynamics of smallworld networks," Nature, pp. 440-442, 1998.

[7] A. Barabasi and R. Albert, "Emergence of scaling in random networks," Science, vol. 286, no. 5439, pp. 509-512, 1999.

[8] R. Albert, H. Jeong, and A.-L. Barabási, "Error and attack tolerance of complex networks," Nature, vol. 406, no. 6794, pp. 378-382, 2001.
[9] F. Xue, E. Bompard, T. Huang, L. Jiang, S. Lu, and H. Zhu, "Interrelation of structure and operational states in cascading failure of overloading lines in power grids," Physica A: Statistical Mechanics and Its Applications, vol. 482, pp. 728-740, 2017.

[10] W. Zhang, W. Pei, and T. Guo, "An efficient method of robustness analysis for power grid under cascading failure," Safety Science, vol. 64, pp. 121-126, 2014.

[11] C. Yi, Y. Bao, J. Jiang, and Y. Xue, "Modeling cascading failures with the crisis of trust in social networks," Physica A: Statistical Mechanics and Its Applications, vol. 436, pp. 256-271, 2015.

[12] T. Liu, P. Li, Y. Chen, J. Zhang, and C. T. Bauch, "Community size effects on epidemic spreading in multiplex social networks," PLoS ONE, vol. 11, no. 3, Article ID e0152021, 2016.

[13] J. J. Wu, H. J. Sun, and Z. Y. Gao, "Cascading failures on weighted urban traffic equilibrium networks," Physica A: Statistical Mechanics and Its Applications, vol. 386, no. 1, pp. 407-413, 2007.

[14] L. Zhang, J. Lu, J. Zhou, J. Zhu, Y. Li, and Q. Wan, “Complexities' day-to-day dynamic evolution analysis and prediction for a Didi taxi trip network based on complex network theory," Modern Physics Letters B, vol. 32, no. 09, Article ID 1850062, 2018.

[15] Z. Neal, "The devil is in the details: Differences in air traffic networks by scale, species, and season," Social Networks, vol. 38, no. 1, pp. 63-73, 2014.

[16] M. Zanin and F. Lillo, "Modelling the air transport with complex networks: a short review," The European Physical Journal Special Topics, vol. 215, no. 1, pp. 5-21, 2013.

[17] S. V. Buldyrev, R. Parshani, G. Paul, H. E. Stanley, and S. Havlin, "Catastrophic cascade of failures in interdependent networks," Nature, vol. 464, no. 7291, pp. 1025-1028, 2010.

[18] L.-G. Mattsson and E. Jenelius, "Vulnerability and resilience of transport systems - A discussion of recent research," Transportation Research Part A: Policy and Practice, vol. 81, pp. 16-34, 2015.

[19] A. Reggiani, P. Nijkamp, and D. Lanzi, "Transport resilience and vulnerability: the role of connectivity," Transportation Research Part A: Policy and Practice, vol. 81, no. 11, pp. 4-15, 2015.

[20] B. Berche, C. Von Ferber, T. Holovatch, and Y. Holovatch, "Resilience of public transport networks against attacks," The European Physical Journal B, vol. 71, no. 1, pp. 125-137, 2009.

[21] G. Leu, H. Abbass, and N. Curtis, "Resilience of ground transportation networks: A case study on Melbourne," in Proceedings of the 33rd Australasian Transport Research Forum, ATRF 2010, Canberra, Australia, October 2010.

[22] Y. Yang, Y. Liu, M. Zhou, F. Li, and C. Sun, "Robustness assessment of urban rail transit based on complex network theory: A case study of the Beijing Subway," Safety Science, vol. 79, pp. 149-162, 2015.

[23] Y. Gu and C. Li, "The robustness of urban rail transit network based on complex network theory," in Proceedings of the $20163 \mathrm{rd}$ International Conference on Materials Engineering, Manufacturing Technology and Control, Taiyuan, China, Feburary 2016.

[24] D. Sun and S. Guan, "Measuring vulnerability of urban metro network from line operation perspective," Transportation Research Part A: Policy and Practice, vol. 94, pp. 348-359, 2016.

[25] B. Deng, G. Chao, and Z. L. Zhang, "Analysis of robustness of bus and subway interdependent network based on the complex network theory," Journal of Southwest China Normal University, vol. 42, no. 5, pp. 22-27, 2017.

[26] A. Reggiani, "Network resilience for transport security: Some methodological considerations," Transport Policy, vol. 28, pp. 63-68, 2013. 
[27] S. Wandelt, X. Sun, and X. Cao, "Computationally efficient attack design for robustness analysis of air transportation networks," Transportmetrica A: Transport Science, vol. 11, no. 10, pp. 939-966, 2015.

[28] X. Sun, S. Wandelt, and X. Cao, "On node criticality in air transportation networks," Networks and Spatial Economics, vol. 17, no. 11, pp. 1-25, 2017.

[29] X. Sun, V. Gollnick, and S. Wandelt, "Robustness analysis metrics for worldwide airport network: a comprehensive study," Chinese Journal of Aeronautics, vol. 30, no. 2, pp. 500-512, 2017.

[30] A. Cardillo, M. Zanin, J. Gómez-Gardeñes, M. Romance, A. J. García del Amo, and S. Boccaletti, "Modeling the multi-layer nature of the European Air Transport Network: Resilience and passengers re-scheduling under random failures," The European Physical Journal Special Topics, vol. 215, no. 1, pp. 23-33, 2013.

[31] E. Rodríguez-Núñez and J. C. García-Palomares, "Measuring the vulnerability of public transport networks," Journal of Transport Geography, vol. 35, pp. 50-63, 2014.

[32] L. Zhang, J. Lu, M. Long, S.-B. Li, and J. Zhou, "A cascading failures perspective based mesoscopic reliability model of weighted public transit network considering congestion effect and user equilibrium evacuation," Mathematical Problems in Engineering, vol. 2018, Article ID 9292375, 13 pages, 2018.

[33] O. Cats and E. Jenelius, "Dynamic vulnerability analysis of public transport networks: mitigation effects of real-time information," Networks and Spatial Economics, vol. 14, no. 3-4, pp. 435-463, 2014.

[34] V. Latora and M. Marchiori, "Is the Boston subway a smallworld network?" Physica A: Statistical Mechanics and Its Applications, vol. 314, no. 1, pp. 109-113, 2002.

[35] K. A. Seaton and L. M. Hackett, "Stations, trains and smallworld networks," Physica A: Statistical Mechanics and Its Applications, vol. 339, no. 3-4, pp. 635-644, 2004.

[36] J. Wu, Z. Gao, H. Sun, and H. Huang, "Urban transit system as a scale-free network," Modern Physics Letters B, vol. 18, no. 19-20, pp. 1043-1049, 2004.

[37] C. V. Ferber, Y. Holovatch, and V. Palchykov, "Scaling in public transport networks," Condensed Matter Physics, vol. 8, no. 1, pp. 225-234, 2005.

[38] J. Sienkiewicz and J. A. Hołyst, "Statistical analysis of 22 public transport networks in Poland," Physical Review E: Statistical, Nonlinear, and Soft Matter Physics, vol. 72, no. 4, Article ID 046127, 2005.

[39] J. S. Zhao, Z. R. Di, and D. H. Wang, "Empirical research on public transport network of Beijing," Complex Systems and Complexity Science, vol. 2, no. 2, pp. 45-48, 2005.

[40] C. V. Ferber, T. Holovatch, Y. Holovatch, and V. Palchykov, "Network harness: metropolis public transport," Physica A: Statistical Mechanics and Its Applications, vol. 380, no. 1, pp. 585591, 2007.

[41] X. Xu, J. Hu, F. Liu, and L. Liu, "Scaling and correlations in three bus-transport networks of China," Physica A: Statistical Mechanics and Its Applications, vol. 374, no. 1, pp. 441-448, 2007.

[42] Y. Z. Chen, N. Li, and D. R. He, "A study on some urban bus transport networks," Physica A: Statistical Mechanics \& Its Applications, vol. 376, no. 1, pp. 747-754, 2007.

[43] H. Lu and Y. Shi, "Complexity of public transport networks," Tsinghua Science and Technology, vol. 12, no. 2, pp. 204-213, 2007.

[44] Y. Qin, N. Zhang, and L. I. Ji-Ming, "Topological analysis of shanghai weighted bus-stop network," Journal of Guangxi Normal University, vol. 26, no. 2, pp. 14-17, 2008.
[45] X. Y. Yan and M. S. Wang, "Topological Properties of Public Transport Networks: A Temporal Perspective," in Proceedings of the Ninth International Conference of Chinese Transportation Professionals (ICCTP), pp. 1-6, Harbin, China, 2009.

[46] S. Ren-cheng and S. Feng-jing, "Complexity of qingdao/s public transport line network," Complex Systems \& Complexity Science, vol. 6, no. 3, pp. 63-68, 2009.

[47] C. V. Ferber, T. Holovatch, Y. Holovatch, and V. Palchykov, "Public transport networks: empirical analysis and modeling," The European Physical Journal B, vol. 68, no. 2, pp. 261-275, 2009.

[48] B. Berche, C. Von Ferber, and T. Holovatch, "Network harness: Bundles of routes in public transport networks," in Proceedings of the 3rd Conference on Statistical Physics. Dedicated to the 100th Anniversary of Mykola Bogolyubov, pp. 3-12, Ukraine, June 2009.

[49] H. Soh, S. Lim, T. Zhang, X. Fu, G. K. K. Lee, T. G. G. Hung et al., "Weighted complex network analysis of travel routes on the Singapore public transportation system," Physica A: Statistical Mechanics and Its Applications, vol. 389, no. 24, pp. 5852-5863, 2010.

[50] B. Wang, K. E. Honghong, and T. Jiang, "Modeling and characteristics analysis of bus transport network of Hangzhou based on complex network theory," Engineering Journal of Wuhan University, vol. 44, no. 3, pp. 404-408, 2011.

[51] X.-H. Yang, G. Chen, B. Sun, S.-Y. Chen, and W.-L. Wang, "Bus transport network model with ideal n-depth clique network topology," Physica A: Statistical Mechanics and Its Applications, vol. 390, no. 23-24, pp. 4660-4672, 2011.

[52] Y. Sui, F. J. Shao, R. C. Sun, and S. J. Li, "Space evolution model and empirical analysis of an urban public transport network," Physica A: Statistical Mechanics \& Its Applications, vol. 391, no. 14, pp. 3708-3717, 2012.

[53] X. Zheng, J. P. Chen, J. L. Shao, and L. D. Bie, "Analysis on topological properties of Beijing urban public transit based on complex network theory," Acta Physica Sinica, vol. 61, no. 19, pp. 190379-190510, 2012.

[54] J. Zheng, J. Chen, and Y. Long, "Shortest path algorithm based on the weighted distance in complex bus transfer network," Urban Transport of China, no. 6, pp. 86-89, 2012.

[55] L. Guo, Y. Zhu, Z. Luo, and W. Li, "The scaling of several public transport networks in China," Fractals-complex Geometry Patterns \& Scaling in Nature \& Society, vol. 21, no. 2, 2013.

[56] A. L. Huang, W. Guan, B. H. Mao, and G. Z. Zang, "Statistical analysis of weighted complex network in Beijing Public Transit Routes System based on passenger flow," Journal of Transportation Systems Engineering \& Information Technology, vol. 13, no. 6, pp. 198-204, 2013.

[57] B. Leng, X. Zhao, and Z. Xiong, "Evaluating the evolution of subway networks: evidence from Beijing subway network," $E P L$ (Europhysics Letters), vol. 105, no. 5, Article ID 58004, pp. 1-6, 2014.

[58] R. Ding, N. Ujang, H. B. Hamid, and J. Wu, "Complex network theory applied to the growth of Kuala Lumpur's public urban rail transit network," PLoS ONE, vol. 10, no. 10, 2015.

[59] Y. Luo and D.-L. Qian, "Construction of subway and bus transport networks and analysis of the network topology characteristics," Journal of Transportation Systems Engineering and Information Technology, vol. 15, no. 5, pp. 39-44, 2015.

[60] L. Alessandretti, M. Karsai, and L. Gauvin, "User-based representation of time-resolved multimodal public transportation 
networks," Royal Society Open Science, vol. 3, no. 7, Article ID 160156, 2016.

[61] S. D. Dimitrov and A. Ceder, "A method of examining the structure and topological properties of public-transport networks," Physica A: Statistical Mechanics and Its Applications, vol. 451, pp. 373-387, 2016.

[62] L. Xu and X. Liu, "The characteristic analysis of transit network in small cities based on the complex network theory," Journal of Wuhan University of Technology, vol. 40, no. 6, pp. 943-948, 2016.

[63] Q. Xu, B. Mao, and Y. Bai, "Network structure of subway passenger flows," Journal of Statistical Mechanics Theory \& Experiment, vol. 3, Article ID 33404, 2016.

[64] R. D. Regt, C. V. Ferber, Y. Holovatch, and M. Lebovka, Public Transportation in UK Viewed as A Complex Network, 2017.

[65] X. Yang and Z. Chen, "Research on public transport network modeling and its performance with public bicycle sharing system," Journal of Zhejiang University of Technology, vol. 46, no. 1, pp. 27-32, 2018.

[66] H. Zhang, C.-X. Zhuge, X. Zhao, and W.-B. Song, "Assessing transfer property and reliability of urban bus network based on complex network theory," International Journal of Modern Physics C, vol. 29, no. 1, Article ID 1850004, 2018.

[67] L. Zhang, J. Lu, X. Yue, J. Zhou, Y. Li, and Q. Wan, "An auxiliary optimization method for complex public transit route network based on link prediction," Modern Physics Letters B. Condensed Matter Physics, Statistical Physics, Applied Physics, vol. 32, no. 5, Article ID 1850066, 2018.

[68] S. Tanuja, I. W. H. Ho, and K. T. Chi, "Spatial analysis of bus transport networks using network theory," Physica A: Statistical Mechanics \& Its Applications, vol. 502, 2018.

[69] L. Zhang, B.-B. Fu, and Y.-X. Li, "Cascading failure of urban weighted public transit network under single station happening emergency," Procedia Engineering, vol. 137, pp. 259-266, 2016.

[70] Y. Dong, X. Yang, and G. Chen, "Robustness analysis of layered public transport networks due to edge overload breakdown," International Journal of Information Technology \& Computer Science, vol. 6, no. 3, pp. 30-37, 2014.

[71] L. Zhang, B.-B. Fu, and S.-B. Li, "Cascading failures coupled model of interdependent double layered public transit network," International Journal of Modern Physics C, vol. 27, no. 12, Article ID 1650145, 2016.

[72] Y. Yang, A. Huang, and W. Guan, "Statistic properties and cascading failures in a coupled transit network consisting of bus and subway systems," International Journal of Modern Physics B, vol. 28, no. 30, Article ID 1450212, 2014.

[73] A. Huang, H. M. Zhang, W. Guan, Y. Yang, and G. Zong, "Cascading failures in weighted complex networks of transit systems based on coupled map lattices," Mathematical Problems in Engineering, vol. 2015, Article ID 940795, 16 pages, 2015.

[74] B. B. Mandelbrot, The Fractal Geometry of Nature, Birkhäuser Verlag, Basel, Switzerland, 1991.

[75] L. Benguigui, "A fractal analysis of the public transportation system of Paris," Environment and Planning A, vol. 27, no. 7, pp. 1147-1161, 1995.

[76] K. S. Kim, L. Benguigui, and M. Marinov, "The fractal structure of Seoul's public transportation system," Cities, vol. 20, no. 1, pp. 31-39, 2003.

[77] C. V. Ferber and Y. Holovatch, "Fractal transit networks: Selfavoiding walks and Lévy flights," The European Physical Journal Special Topics, vol. 216, no. 1, pp. 49-55, 2013.
[78] J. Wu, Z. Gao, and H. Sun, "Complexity and efficiency of Beijing Transit Network," International Journal of Modern Physics B, vol. 20, no. 15, pp. 21-29, 2006.

[79] T. Wang and W. U. Lin-Li, "Research on invulnerability of urban transit network based on complex network," Application Research of Computers, vol. 27, no. 11, pp. 4084-4086, 2010.

[80] H. Duan, Z. Li, and Y. Zhang, "Robustness Analysis Model of Urban Transit Networks," Journal of South China University of Technology, vol. 38, no. 3, pp. 70-75, 2010.

[81] B. Berche, C. von Ferber, T. Holovatch, and Y. Holovatch, "Transportation network stability: a case study of city transit," Advances in Complex Systems. A Multidisciplinary Journal, vol. 15, no. supp01, Article ID 1250063, 2012.

[82] C. V. Ferber, B. Berche, T. Holovatch, and Y. Holovatch, "A tale of two cities: Vulnerabilities of the London and Paris transit networks," Journal of Transportation Security, vol. 5, no. 3, pp. 199-216, 2012.

[83] L. Wu, Q. Tan, and Y. Zhang, "Network connectivity entropy and its application on network connectivity reliability," Physica A: Statistical Mechanics and Its Applications, vol. 392, no. 21, pp. 5536-5541, 2013.

[84] J. Zhang, M. Zhao, H. Liu, and X. Xu, "Networked characteristics of the urban rail transit networks," Physica A: Statistical Mechanics and Its Applications, vol. 392, no. 6, pp. 1538-1546, 2013.

[85] D. J Sun, Y. Zhao, and Q. Lu, "Vulnerability analysis of urban rail transit networks: A case study of Shanghai, China," Sustainability, vol. 7, no. 6, pp. 6919-6936, 2015.

[86] B.-B. Fu, L. Zhang, S.-B. Li, and Y.-X. Li, "Survivability of public transit network based on network structure entropy," International Journal of Modern Physics C, vol. 26, no. 9, Article ID 1550104, 2015.

[87] H. F. Feng, L. CH, and R. Wang, "Vulnerability study for public transport network of valley city: Case of Lanzhou," Journal of Transportation Systems Engineering \& Information Technology, vol. 16, no. 1, pp. 217-222, 2015.

[88] F. Chen, H. U. Ying-Yue, L. I. Xiao-Hong, and P. W. Chen, "Cascading failures in weighted network of urban rail transit," Journal of Transportation Systems Engineering \& Information Technology, vol. 16, no. 2, pp. 139-145, 2016.

[89] T. Ren, Y. Wang, M. Liu, and Y. Xu, "Analysis of robustness of urban bus network," Chinese Physics B, vol. 25, no. 2, Article ID 020101, pp. 1-12, 2016.

[90] X. Wang, Y. Koç, S. Derrible, S. N. Ahmad, W. J. A. Pino, and R. E. Kooij, "Multi-criteria robustness analysis of metro networks," Physica A: Statistical Mechanics and Its Applications, vol. 474, pp. 19-31, 2017.

[91] X. Wu, H. Dong, K. T. Chi, I. W. H. Ho, and F. C. M. Lau, "Analysis of metro network performance from a complex network perspective," Physica A: Statistical Mechanics \& Its Applications, vol. 492, 2017.

[92] J. Zhang, S. Wang, and X. Wang, "Comparison analysis on vulnerability of metro networks based on complex network," Physica A: Statistical Mechanics and Its Applications, vol. 496, pp. 72-78, 2018.

[93] D. Stauffer, Introduction to Percolation Theory, Taylor \& Francis, Abingdon, UK, 1991.

[94] Y. Tu, "How robust is the Internet?" Nature, vol. 406, no. 6794, pp. 353-354, 2000.

[95] H. Jeong, B. Tombor, R. Albert, Z. N. Oltval, and A.-L. Barabásl, "The large-scale organization of metabolic networks," Nature, vol. 407, no. 6804, pp. 651-654, 2000. 
[96] R. Cohen, K. Erez, D. Ben-Avraham, and S. Havlin, "Resilience of the Internet to random breakdowns," Physical Review Letters, vol. 85, no. 21, pp. 4626-4628, 2000.

[97] D. S. Callaway, M. E. J. Newman, S. H. Strogatz, and D. J. Watts, "Network robustness and fragility: percolation on random graphs," Physical Review Letters, vol. 85, no. 25, pp. 5468-5471, 2000.

[98] M. E. O'Kelly, H. Kim, and C. Kim, "Internet reliability with realistic peering," Environment and Planning B: Planning and Design, vol. 33, no. 3, pp. 325-343, 2006.

[99] J. Ma and L. Yang, "Research on cascading failure in public traffic network in guiyang," Information Technology \& Informatization, vol. 6, pp. 74-75, 2011.

[100] Z. Zou, Y. Xiao, and J. Gao, "Robustness analysis of urban transit network based on complex networks theory," Kybernetes, vol. 42, no. 3, pp. 383-399, 2013.

[101] W. Du, J. Yu, X. An, and C. Ma, "Cascading failures of complex public transit network with multi-links based on CML," Transport Research, vol. 1, no. 6, pp. 14-19, 2015.

[102] T. He, N. Zhu, Z. Hou, and G. Xiong, "A Novel Cascading Failure Model on City Transit Network," in Proceedings of the 2016 6th International Conference on Machinery, Materials, Environment, Biotechnology and Computer, Tianjin, China, June 2016.

[103] T. J. Kim and S. Suh, Advanced Transport and Spatial Systems Models, Springer, New York, NY, USA, 1990.

[104] J. Huang, F. Zhou, and M. Xi, "Calculation Method for Load Capacity of Urban Rail Transit Station considering Cascading Failure," Journal of Advanced Transportation, vol. 2018, Article ID 6318516, 12 pages, 2018.

[105] J. J. Fruin, Pedestrian Planning and Design, Metropolitan Association of Urban Designers \& Environmental Planners, 1971.

[106] L. Sun, Y. Huang, Y. Chen, and L. Yao, "Vulnerability assessment of urban rail transit based on multi-static weighted method in Beijing, China," Transportation Research Part A: Policy and Practice, vol. 108, pp. 12-24, 2018.

[107] A. E. Motter and Y. C. Lai, "Cascade-based attacks on complex networks," Physical Review E: Statistical, Nonlinear, and Soft Matter Physics, vol. 66, no. 2, Article ID 65102, 2002.

[108] X. F. Wang and J. Xu, "Cascading failures in coupled map lattices," Physical Review E: Statistical, Nonlinear, and Soft Matter Physics, vol. 70, no. 2, Article ID 56113, 2004.

[109] R. Pant, K. Barker, F. H. Grant, and T. L. Landers, "Interdependent impacts of inoperability at multi-modal transportation container terminals," Transportation Research Part E: Logistics and Transportation Review, vol. 47, no. 5, pp. 722-737, 2011.

[110] W.-B. Du, X.-L. Zhou, O. Lordan, Z. Wang, C. Zhao, and Y.B. Zhu, "Analysis of the Chinese Airline Network as multilayer networks," Transportation Research Part E: Logistics and Transportation Review, vol. 89, pp. 108-116, 2016.

[111] R. Holden, D. V. Val, R. Burkhard, and S. Nodwell, "A network flow model for interdependent infrastructures at the local scale," Safety Science, vol. 53, pp. 51-60, 2013.

[112] A. Veremyev, A. Sorokin, V. Boginski, and E. L. Pasiliao, "Minimum vertex cover problem for coupled interdependent networks with cascading failures," European Journal of Operational Research, vol. 232, no. 3, pp. 499-511, 2014.

[113] X. Ji, B. Wang, D. Liu, G. Chen, F. Tang, and D. Wei, "Improving interdependent networks robustness by adding connectivity links," Physica A: Statistical Mechanics and Its Applications, vol. 444, pp. 9-19, 2016.
[114] L. Tang, K. Jing, J. He, and H. E. Stanley, "Complex interdependent supply chain networks: cascading failure and robustness," Physica A: Statistical Mechanics and Its Applications, vol. 443, pp. 58-69, 2016.

[115] J. Wang, Y. Li, and Q. Zheng, "Cascading load model in interdependent networks with coupled strength," Physica A: Statistical Mechanics and Its Applications, vol. 430, pp. 242-253, 2015.

[116] D. Zhengcheng, F. Yanjun, and T. Meng, "Review on invulnerability of interdependent networks," Complex Systems and Complexity Science, vol. 3, pp. 30-44, 2017.

[117] Z. Su, L. Li, H. Peng, J. Kurths, J. Xiao, and Y. Yang, "Robustness of interrelated traffic networks to cascading failures," Scientific Reports, vol. 4, article 5413, 2014.

[118] R. G. Morris and M. Barthelemy, “Transport on coupled spatial networks," Physical Review Letters, vol. 109, no. 12, Article ID 128703, 2012.

[119] L. Shen, D. Zhang, Y. Xiang, Z. Wang, and T. Zhang, "Simulation on survivability and cascading failure propagation of urban subway-bus compound network," Journal of Southwest Jiaotong University, vol. 53, no. 1, pp. 156-163, 2018.

[120] J. Jin, J. Zhou, and M. Li, Passenger Flow Forecasting Study of Chengdu City Rapid Rail Transit Construction Planning (20122017), Southwest Jiaotong University, Chengdu, China, 2011.

[121] Y. Moreno, R. Pastor-Satorras, A. Vázquez, and A. Vespignani, "Critical load and congestion instabilities in scale-free networks," EPL (Europhysics Letters), vol. 62, no. 2, pp. 292-298, 2007.

[122] J. Wang, C. Jiang, and J. Qian, "Robustness of interdependent networks with different link patterns against cascading failures," Physica A: Statistical Mechanics and Its Applications, vol. 393, no. 1, pp. 535-541, 2014.

[123] M. Zanin, D. Papo, P. A. Sousa et al., "Combining complex networks and data mining: why and how," Physics Reports, vol. 635, pp. 1-44, 2016. 


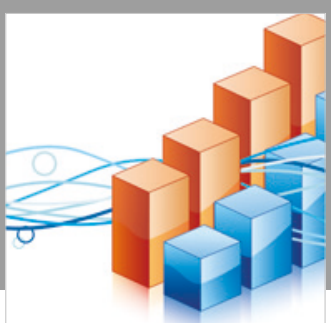

Advances in

Operations Research

\section{-n-m}
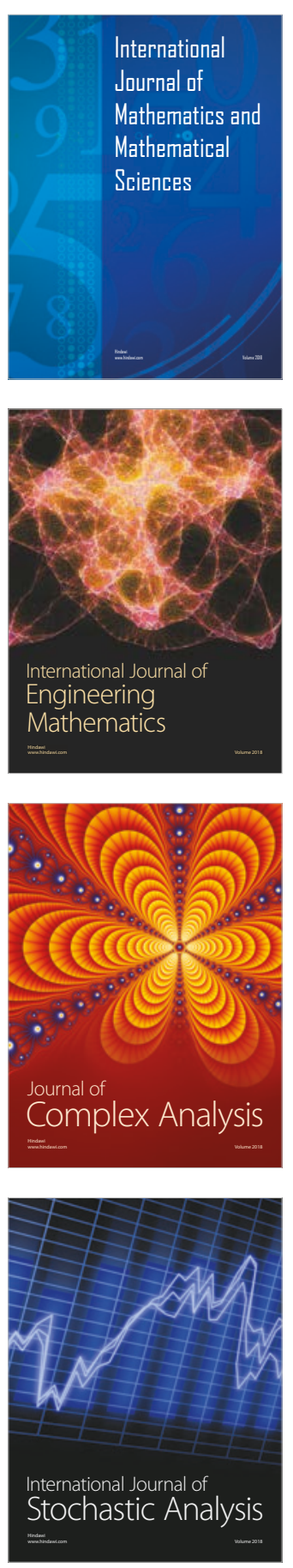
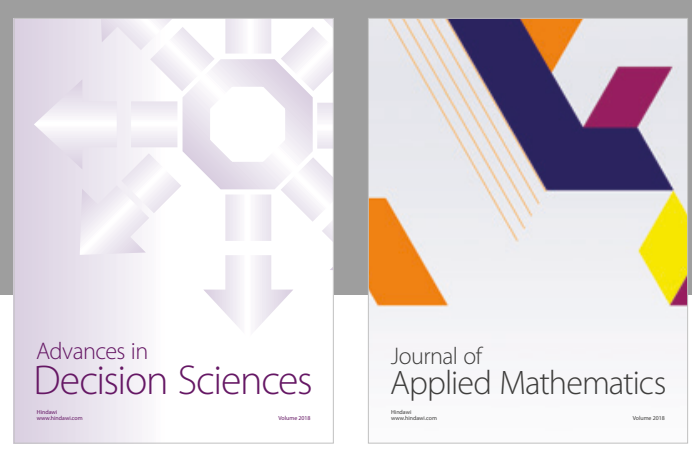

Journal of

Applied Mathematics
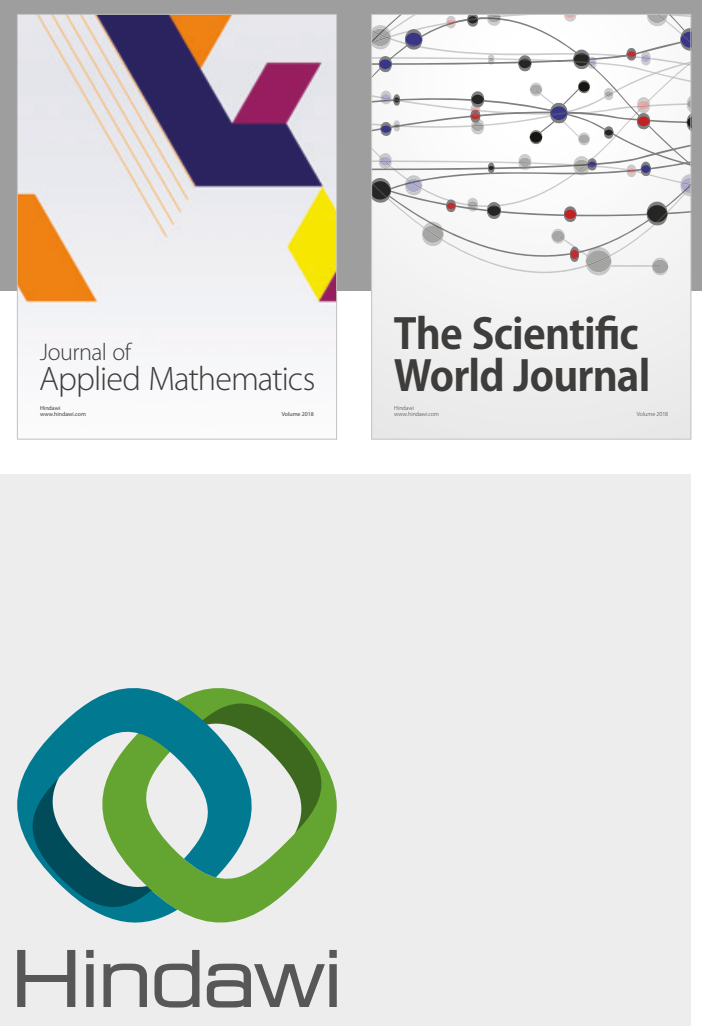

Submit your manuscripts at

www.hindawi.com

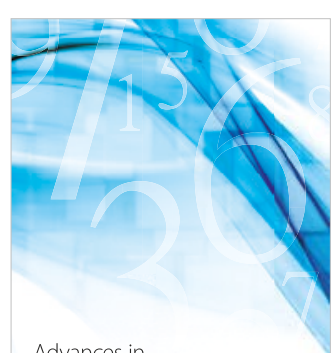

Advances in
Numerical Analysis
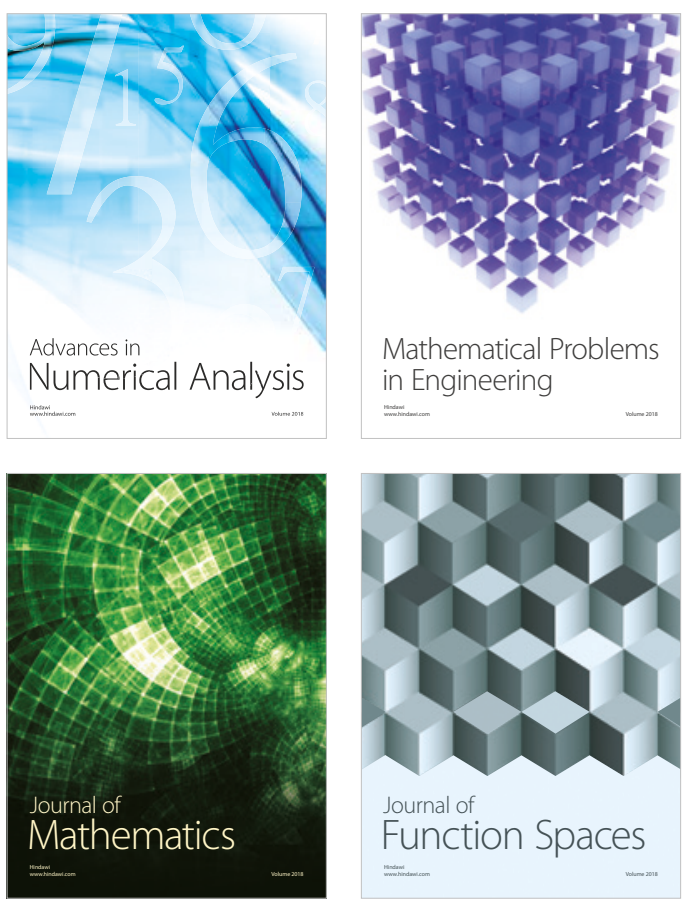

Mathematical Problems in Engineering

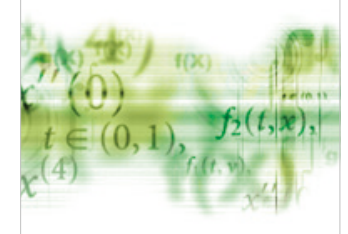

International Journal of

Differential Equations

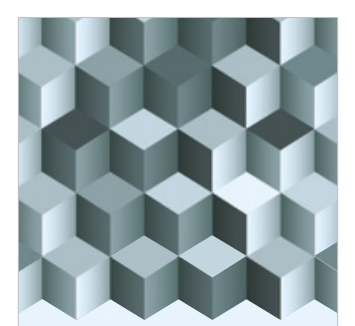

Journal of

Function Spaces
The Scientific

World Journal

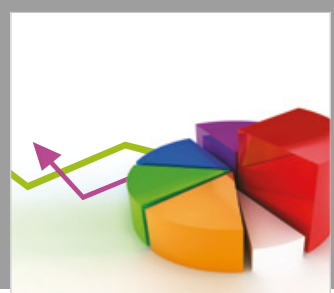

Journal of

Probability and Statistics
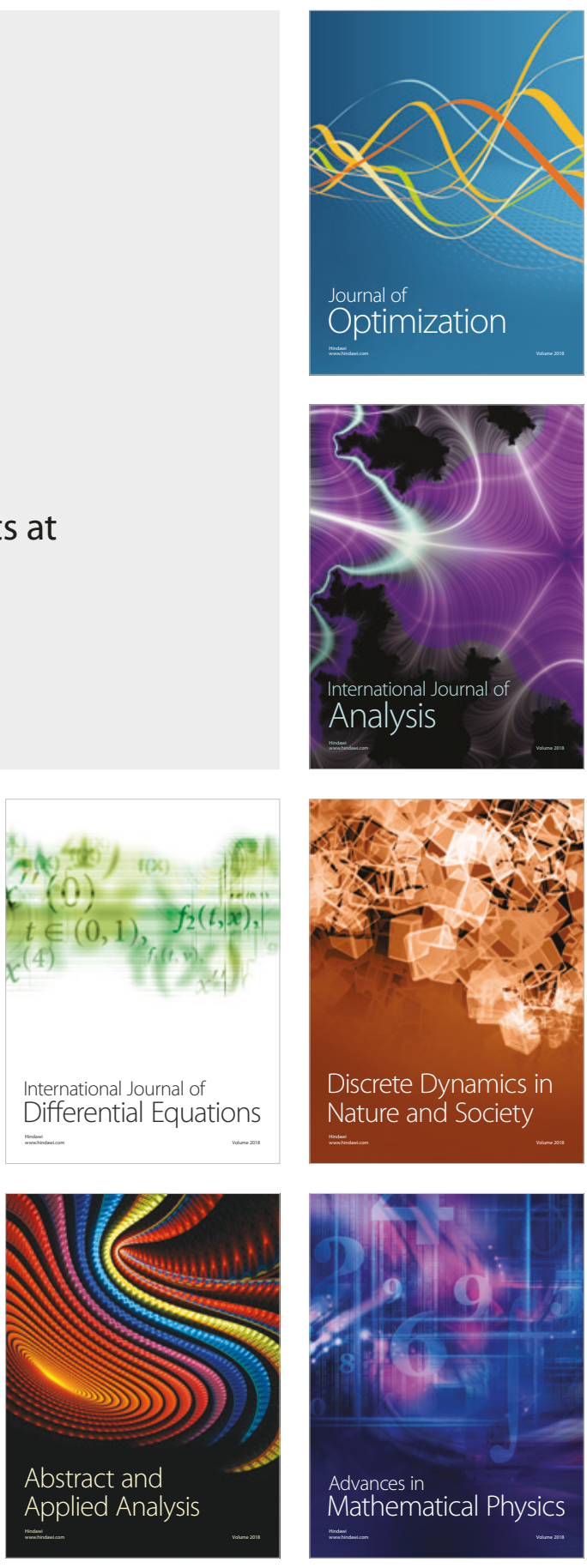